\title{
Lagrangian structures on mapping stacks and semi-classical TFTs
}

\author{
Damien Calaque
}

\begin{abstract}
We extend a recent result of Pantev-Toën-Vaquié-Vezzosi [15], who constructed shifted symplectic structures on derived mapping stacks having a Calabi-Yau source and a shifted symplectic target. Their construction gives a clear conceptual framework for the so-called AKSZ formalism [2].
\end{abstract}

We extend the PTVV construction to derived mapping stacks with boundary conditions, which is required in most applications to quantum field theories (see e.g. the work of CattaneoFelder on the Poisson sigma model [8, 9, and the recent work of Cattaneo-Mnev-Reshetikhin [10, 11]). We provide many examples of Lagrangian and symplectic structures that can be recovered in this way.

We finally give an application to topological field theories (TFTs). We expect that our approach will help to rigorously constuct a 2 dimensional TFT introduced by Moore and Tachikawa in [13. A subsequent paper will be devoted to the construction of fully extended TFTs (in the sense of Baez-Dolan and Lurie [3, 12]) from mapping stacks.

\section{Contents}

Introduction

1 Recollection on shifted symplectic structures 6

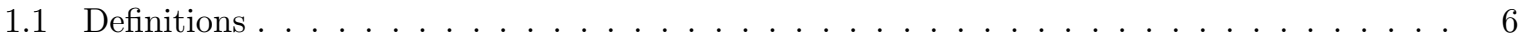

1.2 Examples of shifted symplectic structures . . . . . . . . . . . . 6 6

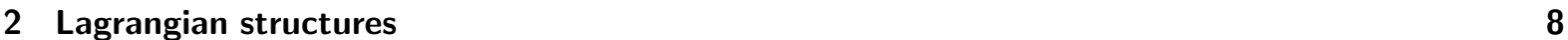

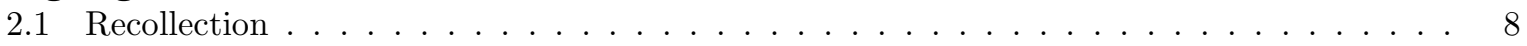

2.2 Examples of Lagrangian structures . . . . . . . . . . . . . . . . . . . . . 10

2.3 Symplectic structures on mapping stacks with boundary conditions . . . . . . . . . . . . 12

3 Recovering usual symplectic and Lagrangian moduli stacks $\quad \mathbf{1 3}$

3.1 Topological context . . . . . . . . . . . . . . . . . . . . . . . . . . . . . . . . . . .

3.2 Algebro-geometric context . . . . . . . . . . . . . . . . . . 14

4 Application: topological field theories from mapping stacks 15

4.1 Classical TFTs from mapping stacks . . . . . . . . . . . . . . . . . . . . . . . .

4.2 Semi-classical TFTs from mapping stacks with $n$-symplectic target . . . . . . . . . . 16

\begin{tabular}{lr} 
Concluding remarks & 18 \\
\hline
\end{tabular} 


\section{Introduction}

\section{Previous works \\ AKSZ construction}

In the seminal paper 2 the authors provide a general procedure that allows one to put many $\sigma$-models in the framework of the BV formalism [5. In the recent papers [10, 11] this construction is extended to the case when the source of the $\sigma$-model has a boundary, and the authors expect that this can further be extended to manifolds with corners and ultimately lead, in the case of topological $\sigma$-models, to fully extended topological field theories as they are defined in [3, 12. The main problems of the AKSZ construction are:

1. it deals with mapping spaces which are infinite dimensional.

2. the mapping spaces considered are formal, in the sense that they actually only capture those maps which are infinitesimally close to the constant ones.

\section{Fully extended TFTs}

Usual topological field theories (TFTs) are axiomatized as symmetric monoidal functors from the category $n$ Cob defined in the following way. Objects are closed manifolds of dimension $n-1$, morphisms are diffeomorphism classes of $n$-dimensional cobordisms between those, and the monoidal structure is given by the disjoint union (notice that there are several variants: unoriented, oriented, framed, ....). Except for the cases $n=1$ and $n=2$ it is very difficult to describe and/or characterise $n$ Cob. There are two successive extensions of $\mathrm{nCob}$ one can consider:

1. in [3] the authors introduce an n-category $n \mathrm{Cob}^{\text {ext }}$ of fully extended cobordisms: objects are closed 0-dimensional manifolds, 1-morphisms are 1-cobordisms between them, 2-morphisms are 2-dimensional cobordisms between the laters, ..., and $n$-morphisms are diffeomorphism classes of cobordisms between $n-1$-dimensional ones. This allows to compute, at least theoretically, the invariant associated to a closed $n$-manifold (viewed as a cobordism between $\emptyset$ and itself) from a triangulation.

2. in [12] it is argued that one should keep track of diffeomorphisms and it is proposed to consider an $(\infty, n)$-category $n \operatorname{Cob}_{\infty}^{\text {ext }}$ of up-and-down extended cobordisms. The meaning of $(\infty, n)$ here is that we have a higher category in which all $k$-morphisms are (weakly) invertible for $k>n$. It is very similar to $\mathrm{nCob}^{\text {ext }}$, except that one keeps track of diffeomorphisms between $\mathrm{n}$-dimensional cobordisms and homotopies between them: $(n+1)$-morphisms are diffeomorphisms, $(n+2)$-morphisms are isotopies between them, $(n+3)$-morphisms are isotopies between isotopies, $\ldots$

The main result of [12] is a characterisation of the framed version $n \operatorname{Cob}_{\infty}^{\text {ext,fr }}$ of $n \operatorname{Cob}_{\infty}^{\text {ext }}$ as the free symmetric monoidal $(\infty, n)$-category generated by a fully dualizable object (a notion we won't explain here, and that should be understood as a strong generalization of finite dimensionality for a vector space in the context of objects in a symmetric monoidal higher category).

\section{PTVV construction}

In a recent paper [15] the AKSZ construction has been interpreted and re-written in the realm of derived (algebraic) geometry (see e.g. [20]). This new approach has several advantages:

1. there is no infinite dimensional complication. There are representability theorems by Lurie and Toën-Vezzosi which guaranty that the mapping stacks we are going to consider are tractable.

2. mapping stacks are no longer formal. From the point-of-view of quantization it gives a hope that we will be able to produce non-perturbative quantum field theories using this formalism.

3. derived geometry is formulated in the language of homotopy theory, which is also the one of modern higher category theory, and is therefore a priori well-suited for fully extended TFTs. 
We briefly summarize the AKSZ/PTVV construction: if $\mathbf{X}$ is a (derived Artin) stack that is "compact" and admits a d-orientation $[\mathbf{X}]$, and if $\mathbf{Y}$ is a stack equipped with an $n$-symplectic structure $\omega$, then $\int_{[X]} e v_{X}^{*} \omega$, where $e v: X \times \operatorname{Map}(X, Y) \rightarrow Y$ is the evaluation map and $\int_{[X]}$ denotes the integration against the fundamental class $[\mathbf{X}]$, is an $(\mathfrak{n}-\mathrm{d})$-symplectic structure on the derived mapping stack $\operatorname{Map}(\mathbf{X}, \mathbf{Y})$. The main examples to keep in mind are when $\mathbf{Y}=\mathrm{BG}$, which is 2-symplectic whenever $\mathrm{G}$ is a reductive algebraic group, and

1. $X=\Sigma_{B}$ is the homotopy type of a compact oriented surface $\Sigma$ : the 0-symplectic structure on $\operatorname{Map}(X, Y)=\operatorname{Loc}_{\mathrm{G}}(\Sigma)$ coincides with the genuine symplectic structure on the moduli space of G-local systems.

2. $\mathbf{X}$ is a $\mathrm{K} 3$ surface: the 0 -symplectic structure on $\operatorname{Map}(\mathbf{X}, \mathbf{Y})=\mathbf{B u n}_{\mathrm{G}}(\mathbf{X})$ coincides with the genuine symplectic structure on the moduli space of G-bundles on $\mathbf{X}$ discovered by Mukai [14].

\section{Motivational conjectures and main results}

\section{Classical fully extended TFTs from mapping stacks}

To any stack $\mathbf{X}$ we associate the $(\infty, 0)$-category (or, $\infty$-groupoid) $\operatorname{Corr}_{(\infty, 0)}(\mathbf{X})$ of stacks over $\mathbf{X}$, where we have discarded all non-invertible morphisms. Assuming we have been able to construct an $(\infty, n)$ category $\operatorname{Corr}_{(\infty, n)}(X)$ for any stack $X$, we then define $(\infty, n+1)$-categories $\operatorname{Corr}_{(\infty, n+1)}(X)$ with objects being stacks over $X$ and having $\operatorname{Corr}_{(\infty, n)}\left(Y_{1} \times{ }_{X}^{h} Y_{2}\right)$ as $(\infty, n)$-category of morphisms from $Y_{1} \rightarrow X$ to $\mathrm{Y}_{2} \rightarrow \mathrm{X}$. If $\mathbf{X}=*$ then $\operatorname{Corr}_{(\infty, n)}(*)$ is symmetric monoidal, with $\otimes:=\times$. We conjecture the following:

Any object $\mathbf{Y}$ in $\operatorname{Corr}_{(\infty, n)}(*)$ is fully dualizable. The induced symmetric monoidal $(\infty, \mathfrak{n})$ functor $\mathrm{Z}_{\mathrm{Y}, \infty}: \mathrm{nCob}_{\infty}^{\text {ext }, \mathrm{fr}} \longrightarrow \operatorname{Corr}_{(\infty, \mathrm{n})}(*)$ can be realized as $\mathbf{M a p}\left((-)_{\mathrm{B}}, \mathrm{Y}\right)$ and factors through $\mathrm{n} \mathrm{Cob}_{\infty}^{\text {ext }}$ (roughly, it doesn't depend on framings).

In the present paper we only consider non fully extended TFTs. We show in Subsection 4.1 that $\operatorname{Map}\left((-)_{\mathrm{B}}, \mathrm{Y}\right)$ produces an honest $\mathrm{n}$-dimensional TFT $z_{\mathrm{Y}}: \mathrm{nCob} \rightarrow$ Corr, where Corr is a genuine category of correspondences obtained by "truncating" $\operatorname{Corr}_{(\infty, n)}(*)$ both above and below: objects in Corr are the $(n-1)$-endomorphisms of the unit in $\operatorname{Corr}_{(\infty, n)}(*)$ (namely, stacks), and morphisms are equivalence classes of $n$-morphisms (i.e. correspondences).

\section{Semi-classical fully extended TFTs from mapping stacks}

We now put a bit of "symplectic flavor" in the above construction. Before doing so let us recall from [15] that there is a notion of Lagrangian structure for a map $f: Y \rightarrow X$ when $X$ is $n$-symplectic. One observes that an $n$-symplectic structure on a stack $\mathbf{X}$ is the same as a Lagrangian structure on the map $\mathbf{X} \rightarrow *_{(n+1)}$, where $*_{(n+1)}$ denotes the point with its canonical $(n+1)$-symplectic structure. Given a k-symplectic stack $\mathbf{X}$ we can define an $(\infty, 0)$-category $\operatorname{Lag}_{(\infty, 0)}(\mathbf{X})$ of maps $\mathbf{Y} \rightarrow \mathbf{X}$ equipped with a Lagrangian structure. Assuming we have been able to construct an $(\infty, \mathfrak{n})$-category $\operatorname{Lag}_{(\infty, n)}(\mathbf{X})$ for any k-symplectic stack $\mathbf{X}$ (and any $k$ ), we then define $(\infty, n+1)$-categories $\operatorname{Lag}_{(\infty, n+1)}(\mathbf{X})$ with objects being morphisms $\mathbf{Y} \rightarrow \mathbf{X}$ equipped with a Lagrangian structure and having $\operatorname{Lag}_{(\infty, n)}\left(\mathbf{Y}_{1} \times{ }_{\mathbf{X}}^{\mathrm{h}} \mathbf{Y}_{2}\right)$ as $(\infty, n)$ category of morphisms from $Y_{1} \rightarrow X$ to $Y_{2} \rightarrow X$. Here we use the fact from [15] that the homotopy fiber product of two Lagrangian morphisms to a given $k$-symplectic stack is $(k-1)$-symplectic. As before, if $\mathbf{X}=*(\mathrm{k})$ then $\operatorname{Lag}_{(\infty, n)}(*(\mathrm{k}))$ is symmetric monoidal with $\otimes:=\times$. We conjecture the following:

Any object $\mathrm{Y}$ in $\operatorname{Lag}_{(\infty, n)}\left(*_{(\mathrm{k})}\right)$ is fully dualizable. The induced symmetric monoidal $(\infty, \mathrm{n})$ -

functor $\mathcal{Z}_{Y_{, \infty}}^{\text {or }}: \mathrm{nCob}_{\infty}^{\text {ext, fr }} \longrightarrow \operatorname{Lag}_{(\infty, n)}(*(\mathrm{k}))$ can be realized as $\operatorname{Map}\left((-)_{\mathrm{B}}, \mathrm{Y}\right)$ and factors through $\mathrm{nCob} b_{\infty}^{\text {ext,or }}$ (roughly, it only depends on the orientation).

Again, in this paper we only consider non fully extended TFTs. We show in Subsection 4.2 that $\operatorname{Map}\left((-)_{\mathrm{B}}, \mathrm{Y}\right)$ produces an honest $\mathrm{n}$-dimensional oriented TFT $z_{Y}^{\text {or }}: \mathrm{nCob}{ }^{\text {or }} \rightarrow \mathrm{LagCorr}_{n-k}$, where LagCorr $_{n-k}$ is a genuine category of Lagrangian correspondences between $(n-k)$-symplectic stacks obtained by "truncating" $\operatorname{Lag}_{(\infty, n)}\left(*_{(k)}\right)$ both above and below: objects in $\operatorname{LagCorr}_{n-k}$ are the $(n-1)-$ endomorphisms of the unit in $\operatorname{Lag}_{(\infty, n)}\left(*_{k}\right)$ ) (namely, $(n-k)$-symplectic stacks), and morphisms are equivalence classes of $\mathrm{n}$-morphisms (Lagrangian correspondences).

Our proof mainly relies on the following result (see Theorem [2.9): 
If $\Sigma$ is a compact $(\mathrm{d}+1)$-manifold with boundary $\partial \Sigma$, then the restriction morphism $\operatorname{Map}\left((\Sigma)_{\mathrm{B}}, \mathrm{Y}\right) \longrightarrow \operatorname{Map}\left((\partial \Sigma)_{\mathrm{B}}, \mathrm{Y}\right)$ has a natural Lagrangian structure. This is a consequence of a more general fact: if $\mathrm{f}: \mathbf{X} \rightarrow \mathbf{X}^{\prime}$ is a morphism between "compact" stacks together with a relative d-orientation then the morphism $\operatorname{Map}\left(\mathbf{X}^{\prime}, \mathbf{Y}\right) \stackrel{f^{*}}{\longrightarrow} \operatorname{Map}(\mathbf{X}, \mathbf{Y})$ is naturally equipped with a Lagrangian structure.

Let us give three examples of Lagrangian structures arrizing in this way:

1. if $\Sigma=\bullet \bullet$, with $\partial \Sigma=\bullet \bullet$, then $z_{Y}^{\text {or }}(\Sigma)$ is the diagonal map $\mathbf{Y} \rightarrow \mathbf{Y} \times \overline{\mathbf{Y}}$ equipped with its standard Lagragian structure (here $\bar{Y}$ means that we equip $Y$ with the opposite shifted symplectic structure).

2. if $\operatorname{dim}(\Sigma)=l$ and $\partial \Sigma=\emptyset$ then $z_{\mathrm{Y}}^{\mathrm{or}}(\Sigma)$ is the morphism $\operatorname{Map}\left((\Sigma)_{\mathrm{B}}, Y\right) \rightarrow *_{(k-l)}$ and the Lagrangian structure on it is precisely the $(k-l-1)$-symplectic structure on the mapping stack obtained via PTTV.

3. if $\mathbf{X}^{\prime}$ is a Fano three-fold with smooth anticanonical divisor $\mathbf{X}$ and $\mathbf{Y}=\mathbf{B G}$, G being a reductive algebraic group, then we recover that $\mathbf{B u n}_{\mathrm{G}}\left(\mathbf{X}^{\prime}\right) \rightarrow \mathbf{B u n}_{\mathrm{G}}(\mathbf{X})$ has a Lagrangian structure (see e.g. $[22])$.

Before going further and describe the contents of the present paper, let us mention three classical field theories that one can recover in this way:

1. if $n=k=3$ and $Y=B G$ then we get the classical Chern-Simons theory.

2. if $n=3, k=1$ and $Y$ is a genuine smooth symplectic variety, then we get classical Rozansky-Witten theory [16].

3. Conjectural. In 13 the authors define a TFT taking values in a category of symplectic varieties equipped with a Hamiltonian action, and urge mathematicians to construct it rigorously. Our approach seems very well-adapted to attack such a problem.

\section{Description of the paper}

We start in Section 1 with some recollection from [15] on $n$-symplectic structures. We continue in Section 2 with some recollection on Lagrangian structures, and provide new examples of these. In particular, we state and prove our main Theorem on Lagrangian structures on derived mapping stacks (Theorem 2.9). We briefly explain in Section 3 how can one recover already known symplectic and Lagrangian moduli spaces (such as the symplectic structure on G-local systems on punctured surfaces with prescribed conjugacy classes of monodromy around punctures). We prove in Section 4 that mapping stacks with Betti source and $n$-symplectic target define semi-classical topological field theories (semiclassical meaning that they take values in some category of Lagrangian correspondences). We finally conclude the paper with some perspectives and a short discussion of boundary conditions.

\section{Acknowledgements}

I thank Bertrand Töen for many very helpful discussions, Michel Vaquié for his kindness in answering a few basic questions, and Tony Pantev for pointing [13] to me. I also thank Pavel Safronov and the anonymous referee, who pointed a few inaccuracies in a previous version.

I started this project after having heard a very enlightening talk by Alberto Cattaneo. The present paper can be seen as a reformulation of some part of 10, 11 in a totally different language1. My subsequent work will be an attempt to make precise [10, Remark 4.2] and [11, §5.3] using this language.

This work has been partially supported by a grant from the Swiss National Science Foundation (project number 200021_137778).

\footnotetext{
${ }^{1}$ We should emphasize that these references actually deal with more general theories than the ones of AKSZ type.
} 


\section{Notation}

Below are the notation and conventions we use in this paper. They can easily be skipped.

- our models for $(\infty, 1)$-categories are categories with weak equivalences (a-k-a relative categories). We refer to 4] for the details about the homotopy theory of relative categories.

- we write he for the homotopy category of an $(\infty, 1)$-category $\mathcal{C}$.

- there is a notion of weak equivalence between relative categories. Hence we have an $(\infty, 1)$-category of (small2) $(\infty, 1)$-categories.

- Top is a good category of topological spaces, and sSet is the category of simplicial sets. They are weakly equivalent as relative categories. The corresponding $(\infty, 1)$-category will be called the $(\infty, 1)$-category of spaces.

- if $\mathcal{C}$ is an $(\infty, 1)$-category then we write $\operatorname{Map}_{\mathcal{C}}(x, y)$ for the space of morphisms from an object $x$ to another object $y$ in $\mathcal{C}$. Paths in $\operatorname{Map}_{\mathcal{C}}(x, y)$ will be refered to as homotopies between morphisms.

- a morphism in an $(\infty, 1)$-category is called an equivalence if it induces an isomorphism in the homotopy category (i.e. if it is homotopic to a zig-zag of weak equivalences).

- $\Gamma$ is the category of pointed sets of the form $[n]:=\{*, 1, \ldots, n\}$, with morphisms being pointed maps. Given a pointed set $X$ and $x \in X \backslash\{*\}$ we denote by $(x): X \rightarrow[1]:=\{*, 1\}$ the map that sends $x$ to 1 and all the other elements to $*$. More generally we denote a pointed map $f:[n] \rightarrow[\mathrm{m}]$ as an $n$-tuple $\left(f^{-1}(1), \ldots, f^{-1}(m)\right)$ of disjoint subsets of $[m]$.

- a symmetric monoidal $(\infty, 1)$-category is a $\Gamma$-shaped diagram $\mathcal{C}$ of relative categories such that for any object $X$ of $\Gamma$ the product $\prod_{x \in X} \mathcal{C}_{(x)}: \mathcal{C}_{X} \longrightarrow\left(\mathcal{C}_{[1]}\right)^{X}$ is a weak equivalence.

- for $n$ objects $x_{1}, \ldots, x_{n}$ of a symmetric monoidal $(\infty, 1)$-category $\mathcal{C}$ (by which we mean objects of $\left.\mathcal{C}_{[1]}\right)$ we write $x_{1} \otimes \cdots \otimes x_{n}$ for the image through $(1 \cdots n)$ of any object $u$ in $\mathcal{C}_{[n]}$ such that $\mathcal{C}_{(i)}(u)=x_{i}$ for all $i$. This is only defined up to a weak equivalence. We also write $1_{\mathcal{C}}$ for the image of the unique object via $\mathcal{C}_{[0]} \rightarrow \mathcal{C}_{[1]}$.

- an object $x$ is called dualizable if there exists an object $x^{\vee}$ and morphisms $1_{\mathcal{C}} \rightarrow \chi \otimes x^{\vee}$ and $x^{\vee} \otimes x \rightarrow 1_{\mathcal{C}}$ such that the composed maps $x^{\vee} \rightarrow x^{\vee} \otimes\left(x \otimes x^{\vee}\right) \tilde{\sim}\left(x^{\vee} \otimes x\right) \otimes x^{\vee} \rightarrow x^{\vee}$ and $x \rightarrow\left(x \otimes x^{\vee}\right) \otimes x \sim \tilde{\rightarrow} x \otimes\left(x^{\vee} \otimes x\right) \rightarrow x$ are homotopic to identities.

- in order to remain on the safe side $\mathbf{k}$ is a field of characteristic zero (but we can probably allow it to be a commutative ring which is Noetherian and of residual characteristic zero).

- for a dualizable $\mathrm{k}$-module $\mathrm{V}$ we might denote $\mathrm{V}^{*}$ its dual $\mathrm{V}^{\vee}$.

- Cpx is the symmetric monoidal $(\infty, 1)$-category of cochain complexes of $\mathbf{k}$-modules.

- $\mathrm{dSt}_{\mathrm{k}}$ is the symmetric monoidal $(\infty, 1)$-category of derived stacks over $\mathrm{k}$ for the étale topology. The symmetric monoidal structure is closed: for any two stacks X, Y there is a "mapping stack" $\operatorname{Map}(X, Y)$. Given a derived stack $X$ we also have the $(\infty, 1)$-category $d S t / X$ of derived stacks over $\mathbf{X}$.

- if $\mathbf{X}$ is a derived stack then $\mathrm{Q} \operatorname{Coh}(\mathbf{X})$ is the symmetric monoidal $(\infty, 1)$-category of quasi-coherent sheaves on $\mathbf{X}$. We also consider the symmetric monoidal $(\infty, 1)$-category $\operatorname{Sh}(\mathbf{X})$ of $\mathbf{k}_{\mathbf{X}}$-modules.

- a derived Artin stack is a derived stack which is m-geometric (for some $\mathrm{m}$ ) w.r.t. the class of smooth morphisms (see [19]) and which is locally of finite presentation (this is a bit more restrictive than the usual Artin condition). In particular, any derived Artin stack $X$ has a dualizable cotangent complex $\mathbb{L}_{X}$, and thus one can define its tangent complex $\mathbb{T}_{X}:=\mathbb{L}_{X}^{\vee}$.

${ }^{2}$ We will mainly ignore size issues. 


\section{Recollection on shifted symplectic structures}

\subsection{Definitions}

In this Subsection we summarize and follow closely [15, Section 1], to which we refer for the details. All along, $\mathbf{X}$ will be a derived Artin stack. We also provide two new examples of 1 -symplectic structures, appearing naturally in Lie theory.

\subsection{1 $p$-forms and closed $p$-forms}

Let us consider the quasi-coherent weighted sheaf $\Omega_{\mathbf{X}}:=S_{\mathcal{O}_{\mathbf{X}}}\left(\mathbb{L}_{\mathbf{X}}[1]\right)$, where the weight $p$ subsheaf is $\Omega_{\mathbf{X}}^{\mathrm{p}}:=\mathrm{S}_{\mathcal{O}_{\mathbf{X}}}^{\mathrm{p}}\left(\mathbb{L}_{\mathbf{X}}[1]\right)$. The space of $\mathrm{p}$-forms of degree $\mathrm{n}$ is

$$
\mathcal{A}^{\mathrm{p}}(\mathbf{X}, \mathrm{n}):=\operatorname{Map}_{\mathbf{S h}(\mathbf{X})}\left(\mathrm{k}_{\mathbf{X}}, \Omega_{\mathbf{X}}^{\mathrm{p}}[\mathrm{n}-\mathrm{p}]\right) \cong \operatorname{Map}_{\mathbf{Q} \operatorname{Coh}(\mathbf{X})}\left(\mathcal{O}_{\mathbf{X}}, \Omega_{\mathbf{X}}^{\mathrm{p}}[\mathrm{n}-\mathrm{p}]\right) .
$$

Remark 1.1. $\Omega_{X}$ can be identified with the sheaf $\operatorname{ev}(\bullet)_{*} \mathcal{O}_{\mathbf{M a p}\left(\mathrm{B} \mathbb{G}_{a}, X\right)}$, where $\operatorname{ev}(\bullet): \operatorname{Map}\left(\mathrm{B} \mathbb{G}_{\mathrm{a}}, \mathrm{X}\right) \rightarrow \mathrm{X}$ is the evaluation at the canonical point $\bullet \rightarrow B \mathbb{G}_{a}$. The weight is given by the $\mathbb{G}_{m}$-action on $\mathrm{B} \mathbb{G}_{a}$.

From the above Remark we see that $\Omega_{X}$ inherits a weight 1 action of $B \mathbb{G}_{\mathbf{a}}$. In concrete terms it boils down to the action of the de Rham differential, which extends the derivation $\mathcal{O}_{\mathbf{X}} \rightarrow \mathbb{L}_{\mathbf{X}}=\mathbb{L}_{\mathbf{X}}[1][-1]$.

We denote by $\Omega_{X}^{c l}$ the $B \mathbb{G}_{a}$-homotopy fixed points of $\Omega_{X}$ within $\mathbb{G}_{m}-\operatorname{Sh}(\mathbf{X})$, and $\Omega_{X}^{p, c l}$ its weight $p$ subsheaf. The space $\mathcal{A}^{\mathbf{p}, \mathrm{cl}}(\mathbf{X}, \mathbf{n})$ of closed $\mathrm{p}$-forms of degree $\mathrm{n}$ is then $\operatorname{Map}_{\mathbf{S h}(\mathbf{X})}\left(\mathbf{k}_{\mathbf{X}}, \Omega_{\mathbf{X}}^{\mathrm{p}, \mathrm{cl}}[\mathrm{n}-\mathrm{p}]\right)$.

There is an obvious morphism $\mathcal{A}^{\mathrm{p}, \mathrm{cl}}(\mathrm{X}, \mathrm{n}) \rightarrow \mathcal{A}^{\mathrm{p}}(\mathrm{X}, \mathrm{n})$. The image, under this map, of a closed $\mathrm{p}$-form of degree $n$ is called its underlying $p$-form. Given a $p$-form $\omega \in \mathcal{A}^{p}(\mathbf{X}, n)$ of degree $n$ we will call its homotopy fiber $\mathcal{A}^{\mathrm{p}, \mathrm{cl}}(\mathbf{X}, \mathrm{n}) \underset{\mathcal{A}^{\mathrm{p}}(\mathbf{X}, \mathrm{n})}{\stackrel{\mathrm{h}}{\times}}\{\boldsymbol{\omega}\}$ the space of keys of $\boldsymbol{\omega}$.

\subsubsection{Shifted sympletic structures}

A 2-form $\omega: \mathcal{O}_{\mathbf{X}} \rightarrow \mathrm{S}_{\mathcal{O}_{\mathbf{X}}}^{2}\left(\mathbb{L}_{\mathbf{X}}[1]\right)[n-2]$ of degree $\mathrm{n}$ is said non-degenerate if the induced map $\mathbb{T}_{\mathbf{X}} \rightarrow \mathbb{L}_{\mathbf{X}}[\mathrm{n}]$ is an isomorphism in $h Q \operatorname{Coh}(\mathbf{X})$. We denote by $\mathcal{A}^{2}(\mathbf{X}, n)^{\text {nd }}$ the subspace of non-degenerate 2-forms of degree $n$.

Remark 1.2. $\mathcal{A}^{2}(\mathrm{X}, \mathrm{n})^{\mathrm{nd}}$ is a union of connected components in $\mathcal{A}^{2}(\mathbf{X}, \mathrm{n})$, therefore the map $\mathcal{A}^{2}(\mathbf{X}, \mathrm{n})^{\mathrm{nd}} \rightarrow$ $\mathcal{A}^{2}(\mathrm{X}, \mathrm{n})$ is a fibration.

An $n$-symplectic form is a closed 2-form of which the underlying 2-form is non-degenerate. We write $\operatorname{Symp}(\mathbf{X}, \mathbf{n})$ for the space of $n$-symplectic forms, defined as the (homotopy) pull-back

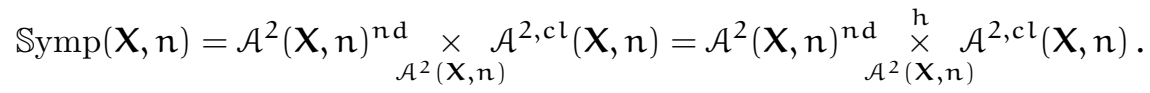

\subsection{Examples of shifted symplectic structures}

In this Subsection we recall the main results from [15, Section 2].

\subsubsection{2-symplectic structures on BG}

Let $G$ be a group scheme and let $X=B G$. Then $\mathbb{L}_{\mathbf{X}}=\mathfrak{g}^{*}[-1]$ and thus $\Omega_{\mathbf{X}}=S\left(\mathfrak{g}^{*}\right)$. Since $\Omega_{\mathbf{X}}$ is concentrated in (cohomological) degree 0 then the action of $B \mathbb{G}_{a}$ on it is trivial.

Remark 1.3. It is a general fact that the homotopy fixed points of the trivial action of $\mathrm{B} \mathbb{G}_{\mathrm{a}}$ on a weighted object $C$ are given by $\bigoplus_{i \geq 0} C[-2 i](i)=C[u]$, where $u$ is a degre 2 variable of weight -1 .

Let us now assume that $\mathrm{G}$ is reductive. We then have:

- $\mathcal{A}^{p}(X, n)=\operatorname{Map}_{G-\bmod }\left(k, S^{p}\left(\mathfrak{g}^{*}\right)[n-p]\right)=\operatorname{Map}_{C p x}\left(k, S^{p}\left(\mathfrak{g}^{*}\right)^{\mathrm{G}}[n-p]\right)$;

- $\mathcal{A}^{p, c l}(X, n)=\operatorname{Map}_{C p x}\left(k, \bigoplus_{i \geq 0} S^{p+i}\left(\mathfrak{g}^{*}\right)^{G}[n-p-2 i]\right)$.

It appears clearly that a 2 -form can only be non-degenerate if its degree is 2 . Therefore we have $\operatorname{Symp}(\mathbf{X}, \mathfrak{n})_{0}=\operatorname{Symp}(\mathbf{X}, 2)_{0}=\pi_{0}(\operatorname{Symp}(\mathbf{X}, 2))=\{$ invariant non-degenerate symmetric bilinear forms on $\mathfrak{g}\}$. 


\subsubsection{A 2-symplectic structure on Perf}

Recall that for any perfect complex $E$ over a stack $\mathbf{X}$ its pull-back through the evaluation map $B \mathbb{G}_{\mathbf{a}} \times$ $\operatorname{Map}\left(\mathrm{B} \mathbb{G}_{a}, X\right) \rightarrow X$ is a $B \mathbb{G}_{a}$-equivariant perfect complex on $B \mathbb{G}_{a} \times \operatorname{Map}\left(B \mathbb{G}_{a}, X\right)$, which we can view as a $B \mathbb{G}_{a}$-equivariant perfect complex $F$ on $\operatorname{Map}\left(B \mathbb{G}_{a}, X\right)$ together with a $B \mathbb{G}_{a}$-equivariant $u: F \rightarrow F$. The trace of $u$ defines a $B \mathbb{G}_{a}$-equivariant function $\mathrm{Ch}(\mathrm{E})$ on $\boldsymbol{M a p}\left(\mathrm{B} \mathbb{G}_{a}, X\right)$, of degree 0 . Its homogeneous part of weight $p$ then defines a closed $p$-form of degree $p$, denoted $\mathrm{Ch}(E)_{p}$, on $\mathbf{X}$.

Applying the above scheme to the tautological perfect complex $\mathcal{E}$ on the stack Perf of perfect complexes, as defined in [19, Definition 1.3.7.5], we get for $p=2$ a closed 2-form $\mathrm{Ch}(\mathcal{E})$ of degree 2 on Perf. According to [15, proof of Theorem 2.13] its underlying 2-form (of degree 2) can be described fairly easily (up to scaling). Recall that $\mathbb{T}_{\text {Perf }}=\mathcal{A}[1]$, where $\mathcal{A}:=\mathcal{H} \operatorname{Hom}(\mathcal{E}, \mathcal{E})$. Then the underlying (degree 2) 2-form of $\mathrm{Ch}(\mathcal{E})_{2}$ is given by

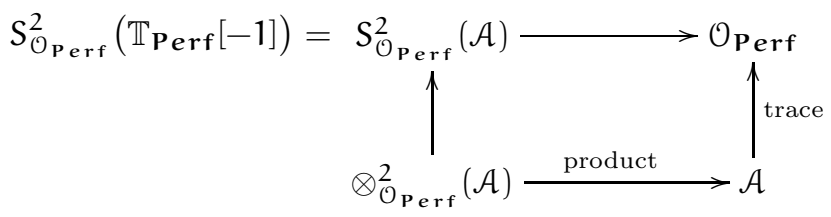

which is obviously non-degenerate. Therefore $\mathrm{Ch}(\mathcal{E})_{2} \in \mathbb{S y m p}(\mathbf{P e r f}, 2)_{0}$.

\subsubsection{A 1-symplectic structure on $\left[\mathfrak{g}^{*} / \mathrm{G}\right]$}

Let $G$ be a group scheme, with Lie algebra $\mathfrak{g}$. We consider the quotient stack $X=\left[\mathfrak{g}^{*} / G\right]$, where $G$ acts on $\mathfrak{g}^{*}$ by the coadjoint action. Then $\mathbb{L}_{\mathbf{X}}=\mathcal{O}_{\mathfrak{g}^{*}} \otimes\left(\mathfrak{g} \oplus \mathfrak{g}^{*}[-1]\right)$ as a $\mathrm{G}$-equivariant quasi-coherent complex on $\mathfrak{g}^{*}$, with differential $d_{\mathfrak{g}}$ being the adjoint action $\mathfrak{g} \ni x \mapsto a d_{x}\left(a d_{x}\right.$ is viewed as a linear function on $\mathfrak{g}^{*}$ with values in $\left.\mathfrak{g}^{*}\right)$.

Observe that the canoncial element in $\mathfrak{g}^{*} \otimes \mathfrak{g}$ defines an element in $\left(S^{2}\left(\mathfrak{g}[1] \oplus \mathfrak{g}^{*}\right)[-1]\right)^{\mathrm{G}}$, and thus a G-equivariant map $\mathcal{O}_{\mathfrak{g}^{*}} \rightarrow \mathcal{O}_{\mathfrak{g}^{*}} \otimes S^{2}\left(\mathfrak{g}[1] \oplus \mathfrak{g}^{*}\right)[-1]=S_{\mathcal{O}_{\mathfrak{g}^{*}}}^{2}\left(\mathbb{L}_{\mathbf{X}}[1]\right)[-1]$ which itself determines a point $\omega$ in $\mathcal{A}^{2}(\mathrm{X}, 1)$. It is obviously non-degenerate (because the canonical element is) and it moreover canonically lifts to a point in $\mathcal{A}^{2, \mathrm{cl}}(\mathrm{X}, 1)$, providing a 1 -symplectic structure on $\mathrm{X}$.

Remark 1.4. Observe that $\mathrm{T}^{*}[1](\mathrm{BG}) \cong\left[\mathfrak{g}^{*} / \mathrm{G}\right]$. What we have just described is nothing but the usual 1-symplectic structure on a shifted cotangent stack (see [15, Proposition 1.21]).

\subsubsection{Shifted symplectic structures on mapping stacks}

Let $\Sigma$ be an $\mathcal{O}$-compact derived stack, following [15, Defintion 2.1]. It ensures the existence of a natural $\mathrm{B} \mathbb{G}_{\mathrm{a}}$-equivariant morphism

$$
\Gamma\left(\Sigma \times \mathbf{X}, \Omega_{\Sigma \times \mathbf{X}}\right) \longrightarrow \Gamma\left(\Sigma, \mathcal{O}_{\Sigma}\right) \otimes \Gamma\left(\mathbf{X}, \Omega_{\mathbf{X}}\right)
$$

within $\mathbb{G}_{\mathrm{m}}-\mathrm{Cpx}$, where $\mathcal{O}_{\Sigma}$ has weight zero and is acted on trivially by $\mathrm{B} \mathbb{G}_{\mathrm{a}}$.

If we further assume that we are given a "fundamental class" $[\Sigma]: \Gamma\left(\Sigma, \mathcal{O}_{\Sigma}\right) \rightarrow \mathbf{k}[-\mathrm{d}]$, then one gets a natural $\mathrm{B} \mathbb{G}_{\mathrm{a}}$-equivariant morphism

$$
\Gamma\left(\Sigma \times \mathbf{X}, \Omega_{\Sigma \times \mathbf{X}}\right) \longrightarrow \Gamma\left(\mathbf{X}, \Omega_{\mathbf{X}}\right)[-\mathrm{d}]
$$

which in particular induces maps $\int_{[\Sigma]}: \mathcal{A}^{\mathfrak{p}(, \mathrm{cl})}(\Sigma \times \mathbf{X}, \mathfrak{n}) \rightarrow \mathcal{A}^{\mathfrak{p}(, \mathrm{cl})}(\mathbf{X}, \mathfrak{n}-\mathrm{d})$. We say that $[\Sigma]$ is a $\mathrm{d}$-orientation if for any perfect complex $\mathrm{E}$ the pairing

$$
\Gamma(\Sigma, E) \otimes \Gamma\left(\Sigma, E^{\vee}\right) \stackrel{\text { duality }}{\longrightarrow} \Gamma\left(\Sigma, \mathcal{O}_{\Sigma}\right) \stackrel{[\Sigma]}{\longrightarrow} \mathbf{k}[-\mathrm{d}]
$$

is non-degenerate. 
Theorem 1.5 (15], Theorem 2.6). For any derived Artin stack $\mathbf{Y}$ and any d-oriented $\mathcal{O}$-compact derived stack $(\Sigma,[\Sigma])$ such that $\operatorname{Map}(\Sigma, \mathbf{Y})$ is itself a derived Artin stack, there is a map

$$
\int_{[\Sigma]} e v_{\Sigma}^{*}(-): \operatorname{Symp}(\mathbf{Y}, \mathfrak{n}) \longrightarrow \operatorname{Symp}(\operatorname{Map}(\Sigma, \mathbf{Y}), \mathfrak{n}-\mathrm{d})
$$

where $\mathrm{e} v_{\Sigma}: \Sigma \times \operatorname{Map}(\Sigma, \mathbf{Y}) \rightarrow \mathbf{Y}$ is the evaluation map.

The procedure that appears in the above result is often refered to as transgression; one actually has transgression maps $\int_{[\Sigma]} e v_{\Sigma}^{*}(-): \mathcal{A}^{p^{(, c l)}}(\mathbf{Y}, \mathfrak{n}) \rightarrow \mathcal{A}^{\mathfrak{p}(, \mathrm{cl})}(\operatorname{Map}(\Sigma, \mathbf{Y}), \mathrm{n}-\mathrm{d})$ for all $\mathrm{p}$. Theorem 1.5] says that the transgression of a non-degenerate 2 -form is non-degenerate.

\subsubsection{1-symplectic structures on $\left[G / G^{a d}\right]$}

Let $G$ be a reductive group scheme and let $X=\left[G / G^{a d}\right]$, where $G^{\text {ad }}$ denotes the action of $G$ on itslef by conjugation. Then $\mathbb{L}_{\mathbf{X}}=\mathrm{T}_{\mathrm{G}}^{*} \oplus \mathcal{O}_{\mathrm{G}} \otimes \mathfrak{g}^{*}[-1]$ as a $\mathrm{G}^{\mathrm{ad}}$-equivariant quasi-coherent complex on $\mathrm{G}$, with differential $d_{L R}$ being adjoint to the infinitesimal action $\mathfrak{g} \rightarrow \mathrm{T}_{\mathrm{G}}$ which sends $x \in \mathfrak{g}$ to the sum $v_{x}:=\chi^{\mathrm{L}}+x^{\mathrm{R}}$ of the corresponding left and right invariant vector fields. Thus $\Omega_{X}$ consists of G-equivariant functions on $\mathfrak{g}$ with values in $\Omega_{G}$, with differential $d_{L R}$ being given by

$$
\mathrm{d}_{\mathrm{LR}}(\mathrm{f})(\mathrm{x})=-\mathfrak{l}_{v_{x}}(\mathrm{f}(\mathrm{x})) \quad\left(\mathrm{f} \in \Omega_{\mathrm{X}}, \mathrm{x} \in \mathfrak{g}\right) .
$$

We now describe an interesting family of 2-forms of degree 1 on $X$. Let $\theta=g^{-1} \mathrm{dg}$ and $\bar{\theta}=\mathrm{dgg}^{-1}$ be the left and right Maurer-Cartan forms on $G$. We then define $\beta:=\frac{1}{2}(\theta+\bar{\theta}) \in\left(\Gamma\left(G, \Omega_{G}^{1}\right) \otimes \mathfrak{g}\right)^{G}$. If we are given a pairing $\langle,\rangle \in S^{2}\left(\mathfrak{g}^{*}\right)^{\mathrm{G}}$ then we get a $\mathrm{G}$-equivariant map $\omega_{0}(x):=\langle\beta, x\rangle$. We claim that $\omega_{0} \in \mathcal{A}^{2}(X, 1)_{0}$ : for any $x \in \mathfrak{g}$,

$$
d_{L R}\left(\omega_{0}\right)(x)=-\left\langle\beta\left(x^{L}+x^{R}\right), x\right\rangle=0 .
$$

One can actually check that if $\langle$,$\rangle is non-degenerate then so is \omega_{0}$.

Remark 1.6. Recall that $\left[\mathrm{G} / \mathrm{G}^{\mathrm{ad}}\right]=\operatorname{Map}\left(\left(\mathrm{S}^{1}\right)_{\mathrm{B}}, \mathrm{BG}\right.$ ). The (degree 1 ) 2-form we've just described is actually the one which is obtained by transgressing the (degree 2) 2-form on BG that is determined by $\langle$,$\rangle . The latter being closed (one should actually rather write closable), so is the former. Below we give$ the "key" that closes it.

Claim 1.7. $\omega_{1}:=\frac{1}{12}\langle\theta,[\theta, \theta]\rangle=\frac{1}{12}\langle\bar{\theta},[\bar{\theta}, \bar{\theta}]\rangle$ closes the 2-form $\omega_{0}$.

Proof. We first have to explain what this statement means. One can observe that the complex of closed $p$-forms is $\Omega_{X}^{p, c l}=\oplus_{i \geq 0} \Omega_{X}^{p+2 i} u^{i}$ with differential being $d_{L R}+u \cdot d$, where $d$ is the usual de Rham differential on $\Omega_{\mathrm{G}}$.

Notice that $\omega_{1}$ is a 3 -form of degree 0 , so that $\omega:=\omega_{0}+u \omega_{1}$ is homogeneous for the homological degree and for the weight. Hence we should prove that $\omega$ is closed, which is a standard calculation in the Cartan model for equivariant cohomology.

\section{Lagrangian structures}

\subsection{Recollection}

In this Subsection we again recollect some definitions and results from [15].

\subsubsection{Isotropic and Lagrangian structures}

Let $(\mathbf{X}, \boldsymbol{\omega})$ be a derived Artin stack equipped with an $n$-symplectic structure, and let $\mathbf{L} \stackrel{\mathrm{f}}{\longrightarrow} \mathbf{X}$ be a morphism of derived stacks. The space Isot $(\mathbf{f}, \boldsymbol{\omega})$ of isotropic structures on $\mathrm{f}$ is the space $\mathcal{A}^{2, \mathrm{cl}}(\mathbf{L}, \mathfrak{n})_{f^{*} \omega, 0}$ of paths from $f^{*} \omega$ to 0 in $\mathcal{A}^{2, c l}(\mathrm{~L}, \mathrm{n})$. 
Let us then define the symplectic orthogonal sheaf $\mathbb{T}_{\mathbf{L}}^{f, \omega}:=\left(f^{*} \mathbb{T}_{\mathbf{X}}\right) \underset{\mathbb{L}_{\mathbf{L}}[\mathrm{n}]}{\stackrel{h}{\times}} 0$ to be the homotopy fiber of the morphism $f^{*} \mathbb{T}_{\mathbf{X}} \rightarrow \mathbb{L}_{\mathbf{L}}[\mathrm{n}]$ given by the underlying 2-form of $\boldsymbol{\omega}$. Notice that any path $\gamma$ from $f^{*} \omega$ to 0 in $\mathcal{A}^{2}(\mathbf{L}, \mathbf{n})$ induces a morphism $\mathbb{T}_{\mathbf{L}} \longrightarrow \mathbb{T}_{\mathbf{L}}^{\mathrm{f}, \omega}$ that makes the following diagram homotopy commutative:

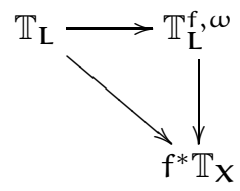

We say that $\gamma$ is non-degenerate if this morphism is an isomorphism in $h \mathrm{QCoh}(\mathbf{L})$.

A Lagrangian structure on $\mathrm{f}$ is an isotropic structure of which the underlying path in $\mathcal{A}^{2}(\mathbf{L}, \mathfrak{n})$ is nondegenerate. We write $\operatorname{Lagr}(f, \boldsymbol{w})$ for the space of Lagrangian structures on $f$, defined as the (homotopy) pull-back

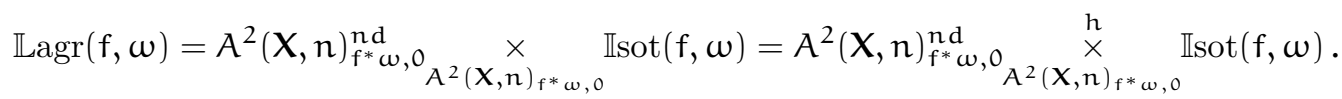

Remark 2.1 (Comparison with Lagrangian structures in [15]). The definition of a Lagrangian structure that is given in [15, Definition 2.8] might look different from ous, but it is equivalent. Namely, an isotropic structure provides a homotopy commutativity data for the following square:

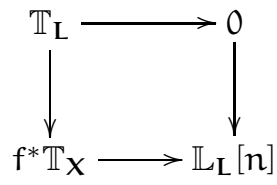

Being Lagrangian is equivalent to homotopy Cartesianity of the above square. This turns out to be equivalent to the induced morphism $\mathbb{T}_{f} \longrightarrow \mathbb{L}_{\mathbf{L}}[n-1]$ being an isomorphism in $h Q \operatorname{Coh}(\mathbf{L})$, where $\mathbb{T}_{f}$ is the relative tangent complex. This last condition is the one which is given in [15, Definition 2.8].

Remark 2.2. Observe that there is yet another formulation of non-degeneracy. An isotropic structure provides a homotopy commutativity data for the following triangle:

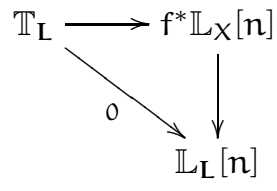

Hence the horizontal map in (2) lifts to a map $\mathbb{T}_{\mathbf{L}} \longrightarrow \mathbb{L}_{\mathrm{f}}[\mathrm{n}-1]$. The isotropic structure is Lagrangian (i.e. non-degenerate) if and only if this map is an isomorphism in $h \mathrm{QCoh}(\mathbf{L})$.

Example 2.3. It is worth noticing that a Lagrangian structure on $\mathbf{X} \rightarrow \bullet_{(\mathrm{n})}$, where $\bullet_{(\mathrm{n})}$ denotes the point $\bullet$ with its canonical (and unique) n-symplectic structure, is nothing else than an $(n-1)$-symplectic structure on $\mathbf{X}$.

\subsubsection{Shifted symplectic structures on derived Lagrangian intersections}

Let $L_{1} \stackrel{f_{1}}{\longrightarrow} X$ and $L_{2} \stackrel{f_{2}}{\longrightarrow} X$ be morphisms of derived Artin stacks, and assume that $\mathbf{X}$ is equipped with an $n$-symplectic structure $\omega$.

Theorem 2.4 ([15], Theorem 2.10). There is a map

$$
\operatorname{Lagr}\left(f_{1}, \omega\right) \times \mathbb{L a g r}\left(f_{2}, \omega\right) \longrightarrow \operatorname{Symp}\left(\underset{L_{1}}{\stackrel{h}{\times}} \mathbf{L}_{2}, n\right)
$$


We now give an explanation for the above result, which we believe is quite enlighting. First of all one observes that it is sufficient to prove Theorem 2.4 for self-intersections (namely, one considers a component in the self-intersection of $\left.L:=L_{1} \amalg L_{2}\right)$. Then notice that for any morphism $L \rightarrow X$ one has a fiber sequence

$$
\mathcal{A}^{\mathrm{p}, \mathrm{cl}}(\mathrm{X} / \mathrm{L}, \mathrm{n}) \longrightarrow \mathcal{A}^{\mathrm{p}, \mathrm{cl}}(\mathrm{X}, \mathrm{n}) \longrightarrow \mathcal{A}^{\mathrm{p}, \mathrm{cl}}(\mathbf{L}, \mathrm{n})
$$

where $\mathbf{X} / \mathbf{L}=\mathbf{X} \coprod_{\mathbf{L}}^{h} \bullet$. One can show that given an $n$-symplectic structure $\boldsymbol{\omega}$ on $\mathbf{X}$, a Lagrangian structure on $\mathrm{L} \rightarrow \mathrm{X}$ provides a lift of $\omega$ to an $n$-symplectic structure $\widetilde{\omega}$ on $X / L$.

Then recall that $\mathbf{L} \underset{\mathbf{X}}{\stackrel{h}{x}} \mathbf{L}$ can be interpreted as the stack of paths in $\mathbf{X}$ with both ends in $\mathbf{L}$, which may be identified with the stack of pointed loops in $\mathbf{X} / \mathbf{L}$. The stack of pointed loops in $\mathrm{X} / \mathrm{L}$ is finally $(n-1)$-symplectic by a variant Theorem 1.5 .

\subsection{Examples of Lagrangian structures}

The main goal of this subsection is to provide new examples of Lagrangian structures.

\subsubsection{Lagrangian morphisms to $\left[\mathfrak{g}^{*} / \mathrm{G}\right]$}

Let $X$ be an actual smooth scheme equipped with a (left) G-action and a G-equivariant morphism $\mu: X \rightarrow \mathfrak{g}^{*}$, where $G$ is a group scheme with Lie algebra $\mathfrak{g}$. This determines a morphism $[X / G] \stackrel{f}{\longrightarrow}\left[\mathfrak{g}^{*} / G\right]$. We describe in this $\S$ interesting Lagrangian structures on $f$ having some genuine symplectic geometric origin. The 1-symplectic structure we consider on $\left[\mathfrak{g}^{*} / \mathrm{G}\right]=\mathrm{T}^{*}[1](\mathrm{BG})$ is the one described in 1.2 .3 Recall that $\mathbb{L}_{[\mathrm{X} / \mathrm{G}]}=\mathrm{T}_{\mathrm{X}}^{*} \oplus \mathcal{O}_{\mathrm{X}} \otimes \mathfrak{g}^{*}[-1]$ as a G-equivariant quasi-coherent complex on $\mathrm{X}$, with differential $\vec{d}$ being adjoint to the infinitesimal action $\mathfrak{g} \rightarrow T_{X}$ which we denote by $\chi \mapsto \vec{x}$. Thus $\Omega_{[X / G]}$ consists of G-equivariant functions on $\mathfrak{g}$ with values in $\Omega_{X}$, with differential $\vec{d}$ being given by

$$
\overrightarrow{\mathrm{d}}(\mathrm{h})(x)=-\mathrm{l}_{\vec{x}}(\mathrm{f}(\mathrm{x})) \quad\left(\mathrm{h} \in \Omega_{[\mathrm{X} / \mathrm{G}]}, x \in \mathfrak{g}\right) .
$$

Borrowing the notation from $\$ 1.2 .3$, a path from $f^{*} \omega$ to 0 in $\mathcal{A}^{2}([\mathrm{X} / \mathrm{G}], 1)$ is then the same as the data of a 2-form $\gamma$ of degree 0 on $[X / G]$ such that $\vec{d}(\gamma)=-f^{*} \omega$; that is to say a G-invariant 2-form $\gamma$ on $X$ such that $\mathfrak{l}_{\vec{x}} \gamma=\mu^{*} d x$, where we view $x \in \mathfrak{g}$ as a linear function on $\mathfrak{g}^{*}$. The non-degeneracy condition boils down to the usual non-degeneracy of the 2 -form $\gamma$. Moreover, if $\gamma$ is closed for the usual de Rham differential on $\mathrm{X}$ then our path admits a canonical lift to $\mathcal{A}^{2, \mathrm{cl}}([\mathrm{X} / \mathrm{G}], 1)$.

Observe that what we have just described (a genuine non-degenerate closed 2-form $\gamma$ on $X$ such that $\mathfrak{t}_{\vec{x}} \gamma=\mu^{*} \mathrm{~d} x$ ) is nothing else than a moment map (or, Hamiltonian) structure on $\mu$.

\subsubsection{Lagrangian morphisms to $\left[\mathrm{G} / \mathrm{G}^{\mathrm{ad}}\right]$}

Let $X$ be an actual smooth scheme equipped with a (left) G-action and a G-equivariant morphism $\mu: X \rightarrow G$, where $G$ is a reductive group scheme. This determines a morphism $[X / G] \stackrel{f}{\longrightarrow}\left[G / G^{a d}\right]$. As above we now describe some interesting Lagrangian structures on $f$, where the symplectic structure on $\left[\mathrm{G} / \mathrm{G}^{\mathrm{ad}}\right]$ is the one determined by a given invariant symmetric bilinear form $\langle$,$\rangle on \mathfrak{g}$ (see $\left.\$ 1.2 .5\right)$.

Borrowing the notation from the previous $\oint$ as well as from $₫ 1.2 .5$, a path from $\mathrm{f}^{*} \omega_{0}$ to 0 in $\mathcal{A}^{2}([\mathrm{X} / \mathrm{G}], 1)$ is then the same as the data of a 2-form $\gamma$ of degree 0 on $[X / G]$ such that $\vec{d}(\gamma)=-f^{*} \omega_{0}$; that is to say a G-invariant 2-form $\gamma$ on $X$ such that $\iota_{\vec{x}} \gamma=\mu^{*}\langle\beta, x\rangle$.

Claim 2.5. The path is non-degenerate if and only if $\operatorname{ker}(\gamma) \cap \operatorname{ker}(\mathrm{d} \mu)=0$.

Proof. First observe that $\mathbb{T}_{f} \cong \mathbb{T}_{\mu}$ is the two-step G-equivariant complex $\mathrm{T}_{\mathrm{X}} \oplus \mu^{*} \mathrm{~T}_{\mathrm{G}}[-1]$ on $\mathrm{X}$ with differential being $d \mu$. Then recall that $\mathbb{L}_{[X / G]}=T_{X}^{*} \oplus \mathcal{O}_{X} \otimes \mathfrak{g}^{*}[-1]$ with differential $\vec{d}$.

The cochain map $\mathbb{T}_{f} \rightarrow \mathbb{L}_{[X / G]}$ induced by the path (see Remark 2.1) has the following form:

- in degree 0 it is simply the G-equivariant map $\mathrm{T}_{\mathrm{X}} \rightarrow \mathrm{T}_{\mathrm{X}}^{*}$ induced by the G-invariant 2-form $\gamma$,

- in degree 1 it is an isomorphism (essentially given by $\langle\beta,-\rangle$ ). 
Being an isomorphism in degree 1, it is a quasi-isomorphism if and only if its restriction on degree 0 cocycles is injective. This is precisely the condition that $\operatorname{ker}(\gamma) \cap \operatorname{ker}(d \mu)=0$.

Let us finally guess what one could require on $\gamma$ in order to lift it to a path in $A^{2, c l}([X / G], 1)$ from $f^{*} \omega$ to 0: a sufficient requirement is that $d_{X} \gamma=-\mu^{*} \omega_{1}$, where $d$ is the de Rham differential on $X$.

Observe that the data of a genuine invariant 2 -form $\gamma$ on $X$ such that $\iota_{\vec{x}} \gamma=\mu^{*}\langle\beta, x\rangle$ for any $x \in \mathfrak{g}$, $\mathrm{d} \gamma=-\mu^{*} \omega_{1}$, and satisfying the above non-degeneracy condition is precisely what is called a Lie group valued moment map (or, quasi-Hamiltonian) structure on $\mu$ (see [1, Definition 2.2]).

\subsubsection{Lagrangian structures on mapping stacks}

Let $\mathrm{f}: \Upsilon \rightarrow \Sigma$ be a morphism between $\mathcal{O}$-compact derived stacks, and assume that $\Upsilon$ is equipped with a fundamental class $[\Upsilon]: \Gamma\left(\Upsilon, \mathcal{O}_{\Upsilon}\right) \rightarrow \mathbf{k}[-\mathrm{d}]$.

Definition 2.6. The space $\mathbb{B} n d(f,[\Upsilon])$ of boundary structures on $f$ is the space of paths from $f_{*}[\Upsilon]$ to 0 in $\operatorname{Map}\left(\Gamma\left(\Sigma, \mathcal{O}_{\Sigma}\right), \boldsymbol{k}[-\mathrm{d}]\right)$.

Hence any boundary structure on $f$ gives rise to a homotopy between the induced map

$$
\int_{\mathrm{f}_{*}[\Upsilon]}: \mathcal{A}^{\mathrm{p}(, \mathrm{cl})}(\Sigma \times \mathrm{X}, \mathrm{n}) \rightarrow \mathcal{A}^{\mathrm{p}(, \mathrm{cl})}(\mathrm{X}, \mathrm{n}-\mathrm{d})
$$

and the zero map for any derived Artin stack $\mathbf{X}$.

Let $\mathbf{Y}$ be a derived Artin stack with a (closed) $p$-form $\omega$ of degree $n$. Then we have a (closed) $p$-form $\int_{[\curlyvee]} e v_{\curlyvee}^{*} \omega$ of degree $n-d$ on $\operatorname{Map}(\Upsilon, Y)$.

Claim 2.7. There is a path in $\mathcal{A}^{\mathrm{p}(, \mathrm{cl})}(\operatorname{Map}(\Sigma, \mathrm{Y}), \mathrm{n}-\mathrm{d})$ between its pull-back along the morphism rest $: \operatorname{Map}(\Sigma, Y) \rightarrow \operatorname{Map}(\Upsilon, Y)$ and 0 .

Proof. The claim is a consequence of

$$
\operatorname{rest}^{*} \int_{[\curlyvee]} e v_{\curlyvee}^{*} \omega=\int_{[\curlyvee]}(\mathrm{id} \times \text { rest })^{*} e v_{\curlyvee}^{*} \omega=\int_{f_{*}[\curlyvee]} e v_{\Sigma}^{*} \omega,
$$

where the second equality follows from the commutativity of

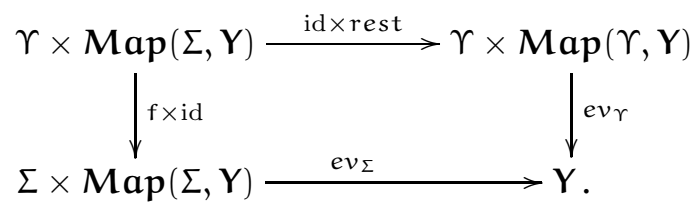

Given a boundary structure on $f: \Upsilon \rightarrow \Sigma$ and a perfect $E$ on $\Sigma$, we get a pairing

$$
\Gamma(\Sigma, E) \otimes \Gamma\left(\Upsilon, f^{*} E^{\vee}\right) \longrightarrow \Gamma\left(\Upsilon, f^{*} E\right) \otimes \Gamma\left(\Upsilon, f^{*} E^{\vee}\right) \stackrel{\text { duality }}{\longrightarrow} \Gamma\left(\Upsilon, \mathcal{O}_{\curlyvee}\right) \stackrel{[\Upsilon]}{\longrightarrow} k[-d]
$$

We write $\Gamma(\Sigma, E)^{\perp}$ for the homotopy fiber of the induced map $\Gamma\left(\Upsilon, f^{*} E^{\vee}\right) \rightarrow \Gamma(\Sigma, E)^{\vee}[-d]$. The path $\gamma$ between $f_{*}[\Upsilon]$ and 0 thus induces a map $\Gamma\left(\Sigma, E^{\vee}\right) \rightarrow \Gamma(\Sigma, E)^{\perp}$ which makes the following diagram homotopy commutative:

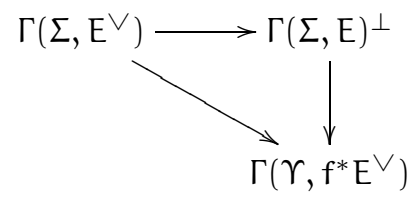

Definition 2.8. We say that a boundary structure $\gamma$ on $f$ is non-degenerate if the induced map $\Gamma\left(\Sigma, E^{\vee}\right) \rightarrow \Gamma(\Sigma, E)^{\perp}$ is an isomorphism in $h C p x$ for any perfect $E$. A relative d-orientation on $f: \Upsilon \rightarrow \Sigma$ is a d-orientation of $\Upsilon$ together with a non-degenerate boundary structure. 
We then have the following "relative" analog of Theorem 1.5

Theorem 2.9. Let $\mathrm{Y}$ be a derived Artin stack equipped with an $\mathrm{n}$-symplectic structure, $\mathrm{f}: \Upsilon \rightarrow \Sigma$ be $a$ morphism of $\mathcal{O}$-compact derived stacks and $[\Upsilon]$ be a d-orientation on $\Sigma$. If the mapping stacks $\mathbf{M a p}(\Sigma, \mathbf{Y})$ and $\operatorname{Map}(\Upsilon, Y)$ are derived Artin stacks then we have a map

$$
\operatorname{Bnd}(\mathrm{f},[\Upsilon]) \longrightarrow \mathbb{I} \text { sot }\left(\text { rest }, \int_{[\curlyvee]} \mathrm{ev} * \boldsymbol{\gamma} \omega\right)
$$

which sends non-degenerate boundary structures to Lagrangian structures.

Here rest $: L=\operatorname{Map}(\Sigma, Y) \rightarrow \operatorname{Map}(\Upsilon, Y)=X$ is again given by composing with $f$.

Proof. We have already seen in Claim 2.7 the existence, for any (closed) p-form $\boldsymbol{\omega}$ of degree $\mathfrak{n}$ on $\mathbf{Y}$, of a map $\mathbb{B}$ nd $(\mathrm{f},[\Upsilon])$ to paths between rest $\int_{[\curlyvee]} e v_{\curlyvee}^{*} \omega$ and 0 in $\mathcal{A}^{p(, c l)}(L, n-d)$.

It remains to show that, whenever $\omega$ is a 2 -form, the above map sends non-degenerate boundary structures to non-degenerate isotropic structures. Note that we already know from Theorem [1.5 that $\tilde{\omega}=\int_{[\curlyvee]} e v_{\curlyvee}^{*} \omega$ is non-degenerate.

For this purpose we describe the map $\mathbb{T}_{\mathbf{L}} \rightarrow \mathbb{T}_{\mathbf{L}}^{r e s t, \tilde{\omega}}$ at a given $A$-point $x: \operatorname{Spec}(A) \rightarrow L$ (i.e. a map $\left.\xi: \Sigma_{A}=\Sigma \times \operatorname{Spec}(A) \rightarrow \mathbf{Y}\right)$. First observe that $\mathbb{T}_{\mathbf{L}, x}=\Gamma\left(\Sigma_{A}, \xi^{*} \mathbb{T}_{\mathbf{Y}}\right)$ and that $\mathbb{T}_{\mathbf{L}, x}^{r e s t, \tilde{\omega}}$ is the homotopy fiber of the map rest ${ }^{*} \mathbb{T}_{\mathbf{X}, x} \longrightarrow \mathbb{L}_{\mathbf{L}, x}[\mathrm{n}-\mathrm{d}]$, which can be decomposed as

$\operatorname{rest}^{*} \mathbb{T}_{\mathbf{X}, x}=\Gamma\left(\Upsilon_{A},(\mathrm{f} \times \mathrm{id})^{*} \xi^{*} \mathbb{T}_{\mathbf{Y}}\right) \stackrel{\sim}{\longrightarrow} \Gamma\left(\Upsilon_{A},(\mathrm{f} \times \mathrm{id})^{*} \xi^{*} \mathbb{L}_{\mathbf{Y}}\right)[\mathrm{n}] \longrightarrow \Gamma\left(\Sigma_{\mathrm{A}}, \xi^{*} \mathbb{L}_{Y}^{\vee}\right)^{\vee}[\mathrm{n}-\mathrm{d}]=\mathbb{L}_{\mathbf{L}, x}[\mathrm{n}-\mathrm{d}]$,

where the first arrow is induced by $\omega$ and the second one is induced by $[\Upsilon]$. Hence $\mathbb{T}_{\mathbf{L}, x}^{\text {rest, } \tilde{\omega}}$ can be identified, using $\omega$, with $\Gamma\left(\Sigma_{A}, \xi^{*} \mathbb{L}_{\mathbf{Y}}^{\vee}\right)^{\perp}[n]$ and the map $\mathbb{T}_{\mathbf{L}, x} \longrightarrow \mathbb{T}_{\mathbf{L}, x}^{\text {rest, } \tilde{\omega}}$ decomposes as

$$
\Gamma\left(\Upsilon_{A}, \xi^{*} \mathbb{T}_{Y}\right) \cong \Gamma\left(\Upsilon_{A}, \xi^{*} \mathbb{L}_{Y}\right)[n] \longrightarrow \Gamma\left(\Sigma_{A}, \xi^{*} \mathbb{L}_{Y}^{\vee}\right)^{\perp}[n] \cong \mathbb{T}_{\mathbf{L}, x}^{r e s t, \tilde{\omega}}
$$

The boundary structure being non-degenerate, it is an isomorphism in $h(A$-mod).

\subsection{Symplectic structures on mapping stacks with boundary conditions}

\subsubsection{Mapping stacks with Lagrangian target}

Let $f: L \rightarrow Y$ be a a morphism of derived Artin stack and let $(\Sigma,[\Sigma])$ be a d-oriented $\mathcal{O}$-compact derived Artin stack such that $\operatorname{Map}(\Sigma, \mathbf{L})$ and $\operatorname{Map}(\Sigma, Y)$ are themselves derived Artin stacks. We have the following obvious extension of Theorem 1.5 (from which we borrow the notation):

Theorem 2.10. If $\omega$ is an $\mathrm{n}$-symplectic structure on $\mathrm{Y}$, then $\int_{[\Sigma]} \mathrm{ev}^{*}(-)$ defines a map

$$
\operatorname{Lagr}(\mathrm{f}, \boldsymbol{\omega}) \longrightarrow \mathbb{L} \text { agr }\left(\mathrm{f} \circ-, \int_{[\Sigma]} e v^{*}(\omega)\right)
$$

\subsubsection{Mapping stacks with boundary conditions}

Let $\mathrm{f}: \Upsilon \rightarrow \Sigma$ and $\mathrm{g}: \mathrm{L} \rightarrow \mathrm{Y}$ be a morphisms of derived Artin stacks. We consider the relative derived mapping stack

$$
\operatorname{Map}(f, g):=\operatorname{Map}(\Upsilon, L) \underset{\operatorname{Map}(\Upsilon, Y)}{\stackrel{h}{\times}} \operatorname{Map}(\Sigma, Y)
$$

and assume that it is a derived Artin stack.

Theorem 2.11. If $\mathrm{f}$ carries a relative $\mathrm{d}$-orientation, $\mathrm{Y}$ carries an $\mathrm{n}$-symplectic structure and $\mathrm{g}$ carries a Lagrangian structure, then $\operatorname{Map}(\mathrm{f}, \mathrm{g})$ has an $(\mathrm{n}-\mathrm{d}-1)$-symplectic structure.

Proof. First of all, the $d$ orientation on $\Upsilon$ and the $n$-symplectic structure on $Y$ produce an $(n-d)$ symplectic structure on $\operatorname{Map}(\Upsilon, Y)$ (by Theorem 1.5). Then the relative d-orientation on $f$ produces a Lagrangian structure on $\operatorname{Map}(\Sigma, \mathbf{Y}$ ) (by Theorem 2.9) and the Lagrangian structure on $\mathrm{g}$ produces a Lagrangian structure on $\operatorname{Map}(\Upsilon, \mathbf{L}$ ) (by Theorem 2.10). Hence, by Theorem 2.4 on derived Lagrangian intersections, $\operatorname{Map}(\mathrm{f}, \mathrm{g})$ inherits an $(\mathrm{n}-\mathrm{d}-1)$-symplectic structure. 


\section{Recovering usual symplectic and Lagrangian moduli stacks}

\subsection{Topological context}

\subsubsection{Boundary structures of Betti type}

Recall that we have a symmetric monoidal $(\infty, 1)$-functor $(-)_{B}:$ Top $\rightarrow d S t_{k}$. For a space $\mathbb{X}$ we have that $\Gamma\left(\mathbb{X}_{\mathrm{B}}, \mathcal{O}_{\mathbb{X}_{\mathrm{B}}}\right)=\mathrm{C}_{\text {sing }}^{*}(\mathbb{X}, \mathbf{k})$. More generally $\mathrm{Q} \operatorname{Coh}\left(\mathbb{X}_{\mathrm{B}}\right)$ is equivalent to the $(\infty, 1)$-category of locally constant sheaves of $k$-modules on $\mathbb{X}$, and $\Gamma\left(\mathbb{X}_{B}, E\right)=\Gamma(\mathbb{X}, E)$ for any object $E$. Note that if $\mathbb{X}$ is compact then $\mathbb{X}_{\mathrm{B}}$ is $\mathcal{O}$-compact.

We now let $M$ be a compact oriented topological manifold of dimension $d+1$ with boundary $\partial M$, and consider the morphism of derived Artin stacks $f: \Upsilon:=(\partial M)_{B} \longrightarrow M_{B}=: \Sigma$ induced by the inclusion of the boundary.

First of all observe that $\partial M$ being closed and oriented, it comes equipped with a fundamental class $[\partial M] \in \mathrm{H}_{\mathrm{d}}(\partial \mathrm{M}, \mathrm{k})$ and hence gives a fundamental class $[\Upsilon]: \Gamma\left(\Upsilon, \mathcal{O}_{\curlyvee}\right) \rightarrow \mathbf{k}[-\mathrm{d}]$ for $\Upsilon$. Poincaré duality guaranties that $[\Upsilon]$ is a d-orientation.

Then the orientation on $M$ provides a relative fundamental class $[M] \in H_{d+1}(M, \partial M, k)$ that is sent to $[\partial M]$ via the boundary map $\mathrm{H}_{\mathrm{d}+1}(\mathrm{M}, \partial \mathrm{M}, \mathbf{k}) \rightarrow \mathrm{H}_{\mathrm{d}}(\partial \mathrm{M}, \mathbf{k})$. Hence约 it determines a path from $\mathrm{f}_{*}[\Upsilon]$ to 0 in $\operatorname{Map}\left(\Gamma\left(\Sigma, \mathcal{O}_{\Sigma}\right), \mathbf{k}[-\mathrm{d}]\right)$. Relative Poincaré duality guaranties that the boundary structure we have just defined on $f: \curlyvee \rightarrow \Sigma$ is non-degenerate.

\subsubsection{Mapping stacks with Betti source}

We borrow the notation from the previous paragraph. Note that if $\mathbb{X}$ is compact then $\operatorname{Map}\left(\mathbb{X}_{B}, \mathbf{Y}\right)$ is a derived Artin stack if $\mathbf{Y}$ is. If moreover $\mathbf{Y}$ carries an $n$-symplectic structure, then Theorem 1.5 tells us that a fundamental class $[\partial M] \in H_{d}(\partial M, k)$ determines an $(n-d)$-symplectic structure on $\operatorname{Map}(\Upsilon, Y)$. Additionally, Theorem 2.9 tells us that a fundamental class $[M] \in H_{d+1}(M, \partial M, k)$ determines a Lagrangian structure on the restriction morphism rest $: \operatorname{Map}(\Sigma, Y) \rightarrow \operatorname{Map}(\Upsilon, Y)$.

In particular, if $G$ is a reductive group and $Y=B G$ we get a $(2-d)$-symplectic structure on $\operatorname{Map}(\Upsilon, B G)=: \operatorname{Loc}_{G}(\partial M)$. Moreover, we have a Lagrangian structure on the restriction morphism $\operatorname{Loc}_{G}(M) \rightarrow \operatorname{Loc}_{G}(\partial M)$.

Example 3.1. When $d=2$, we get in particular a 0 -shifted symplectic structure on the derived moduli stack $\operatorname{Loc}_{G}(\partial M)$ of G-local systems on a compact oriented surface $\partial M$. Recall (see [15, §3.1]) that it induces a genuine symplectic structure on the coarse moduli space $\operatorname{Loc}_{G}^{s}(\partial M)$ of simple G-local systems on $\partial M$. Moreover, the existence of a Lagrangian structure on the restriction map $\operatorname{Loc}_{\mathrm{G}}(M) \rightarrow \operatorname{Loc}_{\mathrm{G}}(\partial M)$ tells us in particular that the regular locus of the subspace in $\operatorname{Loc}_{\mathrm{G}}^{\mathrm{s}}(\partial \mathrm{M})$ consisting of those G-local systems extending to $M$ is a Lagrangian subvariety. This known fact is the starting point of the construction of the Casson invariant.

Example 3.2. If $d=1$ then $\partial M$ is a sum of circles $\left(S^{1}\right)^{n}$ and thus $\operatorname{Map}(\Upsilon, B G)=\left[G / G^{a d}\right]^{\times n}$ is equipped with the 1-symplectic structure of 1.2 .5 and we have a Lagrangian structure on the morphism $\operatorname{Loc}_{G}(M)=\operatorname{Map}(\Sigma, B G) \longrightarrow\left[G / G^{a d}\right]^{\times n}$. Let now $O_{1}, \ldots, O_{n}$ be conjugacy classes in $G$; then the morphism $\left[\mathrm{O}_{1} / \mathrm{G}^{\mathrm{ad}}\right] \times \cdots \times\left[\mathrm{O}_{\mathrm{n}} / \mathrm{G}^{\mathrm{ad}}\right] \longrightarrow\left[\mathrm{G} / \mathrm{G}^{\mathrm{ad}}\right]^{\times n}$ comes equipped with a Lagrangian structure (see 2.2.2). Hence the derived fiber product

$$
\operatorname{Loc}_{G}\left(M ; O_{1}, \ldots, O_{n}\right):=\operatorname{Loc}_{G}(M) \underset{\left[G / G^{a d}\right] \times n}{\stackrel{h}{x}}\left(\left[O_{1} / G^{a d}\right] \times \cdots \times\left[O_{n} / G^{a d}\right]\right)
$$

inherits a 0 -symplectic structure. If $\partial M \neq \emptyset$ then one can show that $\operatorname{Loc}_{G}(M)$ is a smooth DeligneMumford stack. Moreover, for a generic collection $\left(\mathrm{O}_{1}, \ldots, \mathrm{O}_{n}\right)$ of conjugacy classes, the derived fiber product $\operatorname{Loc}_{G}\left(M ; \mathrm{O}_{1}, \ldots, \mathrm{O}_{n}\right)$ is also a smooth Deligne-Mumford stack. We recover in this way the symplectic structure on the moduli space of local systems on a surface with prescribed holonomy along the boundary components (see [1, Section 9] and references therein).

${ }^{3}$ Because $C_{*}(M, \partial M, k)[-1]$ is the homotopy fiber of the map $C_{*}(\partial M, k) \rightarrow C_{*}(M, k)$. 


\subsection{Algebro-geometric context}

\subsubsection{Boundary structures of algebro-geometric type}

Let $\Sigma$ be a geometrically connected smooth proper algebraic variety of dimension $d+1$ together with a smooth d-Calabi-Yau divisor $\Upsilon$ having anticanonical class. We have a fundamental class

$$
[\Upsilon]: \Gamma\left(\Upsilon, \mathcal{O}_{\curlyvee}\right) \longrightarrow \mathrm{H}^{\mathrm{d}}\left(\Upsilon, \mathcal{O}_{\curlyvee}\right)[-\mathrm{d}] \stackrel{\sim}{\longrightarrow} \mathrm{H}^{\mathrm{d}}\left(\Upsilon, \mathcal{K}_{\Upsilon}\right)[-\mathrm{d}] \cong \mathbf{k}[-\mathrm{d}]
$$

where $\mathcal{K}_{\curlyvee}:=\wedge^{\mathrm{d}}\left(\mathrm{L}_{\curlyvee}\right)$ is the canonical sheaf. This is actually a d-orientation by Serre duality.

Then using that $\mathcal{K}_{\Sigma}:=\wedge^{\mathrm{d}+1}\left(\mathrm{~L}_{\Sigma}\right)$ is isomorphic to $\mathcal{O}_{\Sigma}(-\Upsilon)$ we get a relative fundamental class

$$
[\Sigma]: \Gamma\left(\Sigma, \mathcal{O}_{\Sigma}(-\Upsilon)\right) \longrightarrow \mathrm{H}^{\mathrm{d}+1}\left(\Sigma, \mathcal{O}_{\Sigma}(-\Upsilon)\right)[-\mathrm{d}-1] \stackrel{\sim}{\longrightarrow} \mathrm{H}^{\mathrm{d}+1}\left(\Sigma, \mathcal{K}_{\Sigma}\right)[-\mathrm{d}-1] \cong \mathrm{k}[-\mathrm{d}-1]
$$

which lifts $[\Upsilon]$. Namely, if we denote by $f$ the inclusion of $\Upsilon$ into $\Sigma$ then there is a short exact sequence

$$
\mathrm{O} \longrightarrow \mathrm{O}_{\Sigma}(-\Upsilon) \longrightarrow \mathrm{O}_{\Sigma} \longrightarrow \mathrm{f}_{*} \mathrm{O}_{\curlyvee} \longrightarrow 0
$$

such that the induced map $\mathrm{b}: \Gamma\left(\Upsilon, \mathcal{O}_{\curlyvee}\right) \longrightarrow \Gamma\left(\Sigma, \mathrm{O}_{\Sigma}(-\Upsilon)\right)[1]$ makes the following diagram commute:

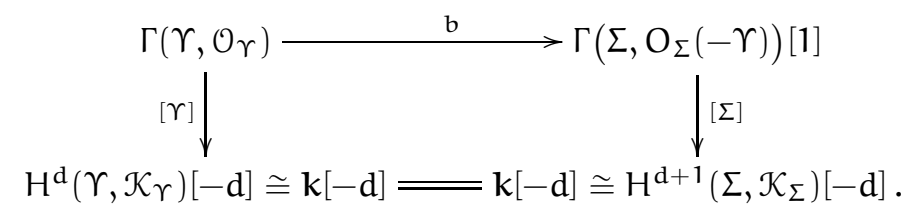

Exactness of (3) tells us in particular that $\Gamma\left(\Sigma, \mathcal{O}_{\Sigma}\right)$ is the homotopy fiber of $b$. Therefore $[\Sigma]$ provides a homotopy between $f_{*}[\Upsilon]$ and 0 in $\operatorname{Map}\left(\Gamma\left(\Sigma, \mathcal{O}_{\Sigma}\right), k[-d]\right)$, that is to say a boundary structure on $f$.

Claim 3.3. This boundary structure is non-degenerate.

Proof. Let $\mathrm{E}$ be a perfect complex on $\Sigma$. We shall prove that the map $\Gamma\left(\Sigma, E^{\vee}\right) \rightarrow \Gamma(\Sigma, E)^{\perp}$ is an isomorphism in $h \mathrm{Cpx}$. Recall that $\Gamma(\Sigma, E)^{\perp}$ is the homotopy fiber of

$$
\Gamma\left(\Upsilon, f^{*} E^{\vee}\right) \cong \Gamma\left(\Upsilon, f^{*} E\right)^{\vee}[-d] \longrightarrow \Gamma(\Sigma, E)^{\vee}[-d]
$$

which is nothing but $\Gamma\left(\Sigma, E \otimes \mathcal{K}_{\Sigma}\right)^{\vee}[-\mathrm{d}-1]$ (again by exactness of (3) ).

One can then easily check that the map $\Gamma\left(\Sigma, E^{\vee}\right) \rightarrow \Gamma\left(\Sigma, E \otimes \mathcal{K}_{\Sigma}\right)^{\vee}[-\mathrm{d}-1]$ provided by the boundary structure is adjoint to the following pairing:

$$
\Gamma\left(\Sigma, E^{\vee}\right) \otimes \Gamma\left(\Sigma, E \otimes \mathcal{K}_{\Sigma}\right) \stackrel{\text { duality }}{\longrightarrow} \Gamma\left(\Sigma, \mathcal{K}_{\Sigma}\right) \stackrel{[\Sigma]}{\longrightarrow} \mathbf{k}[-\mathrm{d}-1] .
$$

Hence the $\operatorname{map} \Gamma\left(\Sigma, E^{\vee}\right) \rightarrow \Gamma(\Sigma, E)^{\perp}$ is an isomorphism in $h C p x$ by Serre duality.

We refer to [21] for a more general statement dealing with the case of a non-smooth divisor.

\subsubsection{Mapping stack with Fano and Calabi-Yau sources}

First observe that any geometrically connected smooth proper algebraic variety $\Sigma$ (over $\mathbf{k}$ ), considered as a derived stack, is $\mathcal{O}$-compact. Then $\operatorname{Map}(\Sigma, Y)$ is a derived Artin stack if $Y$ is. Let us further assume that $\mathrm{Y}$ carries an $\mathrm{n}$-symplectic form.

Using Theorems 1.5 and 2.9, and borrowing the notation from the previous paragraph, we obtain an $(n-d)$-symplectic structure on $\operatorname{Map}(\Upsilon, Y)$ together with a Lagrangian structure on the restriction morphism rest $: \operatorname{Map}(\Sigma, Y) \rightarrow \operatorname{Map}(\Upsilon, Y)$.

In particular, if $\mathrm{G}$ is a reductive group and $\mathrm{Y}=\mathrm{BG}$ we get a $(2-\mathrm{d})$-symplectic structure on $\operatorname{Map}(\Upsilon, Y)=\operatorname{Bun}_{\mathrm{G}}(\Upsilon)$ and a Lagrangian structure on the restriction morphism $\operatorname{Bun}_{\mathrm{G}}(\Sigma) \rightarrow \operatorname{Bun}_{\mathrm{G}}(\Upsilon)$. Similarly, if $\mathbf{Y}=\operatorname{Perf}$ then we get $(2-\mathrm{d})$-symplectic structure on $\operatorname{Perf}(\Upsilon)$ and a Lagrangian structure on the restriction morphism $\operatorname{Perf}(\Sigma) \rightarrow \operatorname{Perf}(\Upsilon)$. 
Example 3.4. If $d=1$ then $\Sigma$ is a del Pezzo surface and $\Upsilon$ is an elliptic curve. Hence $B \mathbf{B n}_{\mathrm{G}}(\Upsilon)$ is 1-symplectic. Consider a semi-stable G-bundle $\mathrm{E}$, which determines a point in $\mathbf{B u n}_{\mathrm{G}}(\Upsilon)$. Formally around that point $\operatorname{Bun}_{\mathrm{G}}(\Upsilon)$ is isomorphic to the neighbourhood of the unit in $\mathrm{H} / \mathrm{H}^{\mathrm{ad}}$, for some reductive subgroup $\mathrm{H} \subset \mathrm{G}$. Hence $\mathrm{E}$ provides us with a Lagrangian morphism $\mathrm{BH} \longrightarrow \mathbf{B u n}_{\mathrm{G}}(\Upsilon)$. Note that the restriction morphism $\operatorname{Bun}_{\mathrm{G}}(\Sigma) \rightarrow \mathbf{B u n}_{\mathrm{G}}(\Upsilon)$ also has a Lagrangian structure. Therefore

$$
\operatorname{Bun}_{\mathrm{G}}(\Sigma ; \Upsilon, E):=\operatorname{Bun}_{\mathrm{G}}(\Sigma) \underset{\operatorname{Bun}_{\mathrm{G}}(\Upsilon)}{\stackrel{\mathrm{h}}{\times}} \mathrm{BH},
$$

which is nothing but the derived moduli stack of $(\Upsilon, E)$-framed G-bundles on $\Sigma$, is 0 -symplectic. When BG is replaced by Perf the genuine symplectic structure that we get on the smooth locus should coincide with the ones defined in [6, 18].

Example 3.5. If $d=2$ then $\Sigma$ is a Fano 3-fold and $\Upsilon$ is a $K 3$ surface. In 15 it is proven that the 0symplectic structure on $\operatorname{Map}(\Upsilon, \operatorname{Perf})=\operatorname{Perf}(\Upsilon)$ induces an actual symplectic structure on the coarse moduli space $\operatorname{Perf}^{s}(\Upsilon)$ of simple perfect complexes on $\Upsilon$. The existence of a Lagrangian structure on $\operatorname{Perf}(\Sigma) \rightarrow \operatorname{Perf}(\Upsilon)$ tells us that the closed subspace of $\operatorname{Perf}^{s}(\Upsilon)$ consisting of restrictions of perfect complexes on $\Sigma$ is Lagrangian (compare with [22, Proposition 2.1]).

\section{Application: topological field theories from mapping stacks}

Let $M$ be a closed oriented topological 3-manifold together with an emmbedded closed oriented surface $S$ that separates it into two part $M_{+}$and $M_{-}: \partial M_{+}=S=\partial \bar{M}_{-}$.

Let $\mathrm{G}$ be a reductive group and observe that we have a weak equivalence of derived Artin stacks

$$
\operatorname{Loc}_{G}(M) \stackrel{\sim}{\longrightarrow} \operatorname{Loc}_{G}\left(M_{+}\right) \underset{\operatorname{Loc}_{G}(S)}{\stackrel{h}{\times}} \operatorname{Loc}_{G}\left(M_{-}\right) .
$$

One can actually prove that it preserves the $(-1)$-symplectic structures on both sides, where the one on the r.h.s. is coming from the derived fiber product of Lagrangian morphisms.

We view the above as an instance of a more general fact that we prove in this Section:

The functor $\operatorname{Map}\left((-)_{\mathrm{B}}, \mathrm{Y}\right)$ defines a topological field theory with values in a suitable category of Lagrangian correspondences whenever $\mathrm{Y}$ admits an $\mathrm{n}$-symplectic structure.

\subsection{Classical TFTs from mapping stacks}

\subsubsection{The cobordism category}

Let $d \geq 0$ be an integer. We define $d C o b$, resp. $d C$ ob ${ }^{\text {or }}$, to be the category with objects being closed differentiable manifolds, resp. oriented differentiable manifolds, and morphisms being diffeomorphism classes of cobordisms, resp. oriented cobordisms. It is a symmetric monoidal category, with monoidal product the disjoint union.

A d-dimensional TFT with values in a symmetric monoidal category $\mathcal{C}$ is a symmetric monoidal functor $\mathrm{dCob} \rightarrow \mathrm{C}$.

\subsubsection{A category of cospans}

We let Cosp be the category with object being compact spaces and $\operatorname{Hom}_{\operatorname{Cosp}}(\mathrm{X}, \mathrm{Y})$ being weak equivalence classes of cospans $\mathrm{X} \rightarrow \mathrm{F} \leftarrow \mathrm{Y}$. Composition of morphisms is given by the homotopy push-out: for $X \rightarrow F \leftarrow Y$ and $Y \rightarrow G \leftarrow Z$, we define $X \rightarrow G \circ F \leftarrow Z$ to be $X \rightarrow F \coprod_{Y}^{h} G \leftarrow Z$. It is symmetric monoidal, with monoidal product the disjoint union (the categorical sum).

For every $\mathrm{d} \geq 0$ there is a symmetric monoidal functor $\mathcal{F}: \mathrm{dCob} \rightarrow \operatorname{Cos} p$ which sends a differentiable manifold to its underlying topological space and a cobordism to the corresponding cospan of spaces. The functoriality of the assignement follows from the fact that inclusions of boundary components in a differentiable manifold are cofibrations (which itself follows from the existence of collars), and guaranties that the ordinary push-out is a homotopy push-out. 


\subsubsection{A category of correspondences}

Let Corr be the category with objects being derived Artin stacks and $H_{\text {Corr }}(\mathbf{X}, \mathbf{Y})$ being weak equivalence classes of correspondences $\mathbf{X} \leftarrow \mathbf{F} \rightarrow \mathrm{Y}$. Composition of morphisms is given by the homotopy fiber product: for $\mathbf{X} \leftarrow \mathbf{F} \rightarrow \mathbf{Y}$ and $\mathbf{Y} \leftarrow \mathbf{G} \rightarrow \mathbf{Z}$, we define $\mathbf{X} \leftarrow \mathbf{G} \circ \mathbf{F} \rightarrow \mathbf{Z}$ to be $\mathbf{X} \leftarrow \underset{\mathrm{Y}}{\mathbf{F}} \underset{\mathbf{G}}{\mathrm{G}} \rightarrow \mathbf{Z}$. It is symmetric monoidal, with monoidal product the Cartesian product.

For any derived Artin stack $Y$ we have a symmetric monoidal functor $\operatorname{Map}\left((-)_{\mathrm{B}}, \mathbf{Y}\right): \operatorname{Cosp} \rightarrow \operatorname{Corr}$. Composed with $\mathcal{F}$ this gives, for every $\mathrm{d} \geq 0$, a d-dimensional TFT $z_{Y}$ with values in Corr.

\subsection{Semi-classical TFTs from mapping stacks with $n$-symplectic target}

\subsubsection{A category of d-oriented cospans}

Let us notice that a compact topological space $\mathbb{X}$ is an oriented Poincaré duality space of dimension $\mathrm{d}$ (we say $\mathrm{d}$-oriented) if and only if the corresponding $\mathcal{O}$-compact derived Artin stack $\mathbb{X}_{\mathrm{B}}$ is $\mathrm{d}$-oriented. Hence, if $\mathbb{X}$ is a $d$-oriented compact space then a relative d-orientation on a map $f: \mathbb{X} \rightarrow \mathbb{X}^{\prime}$ is defined as a non-degenerate boundary structure on $f_{B}: \mathbb{X}_{B} \rightarrow \mathbb{X}_{B}^{\prime}$.

Remark 4.1. Let us give an alternative (and probably easier) description of relative d-orientations. We have a fundamental class $[\mathbb{X}]: C_{\text {sing }}^{*}(\mathbb{X}, \mathbf{k}) \rightarrow \mathbf{k}[-\mathrm{d}]$ and a path from $\mathrm{f}_{*}[\mathbb{X}]$ to 0 in $\mathbb{M a p}\left(C_{\text {sing }}^{*}(\mathbb{X}, \mathbf{k}), \mathbf{k}[-\mathrm{d}]\right)$. Such a path provides, for any perfect local system $E$ on $\mathbb{X}^{\prime}$, a homotopy commutativity data for the following diagram:

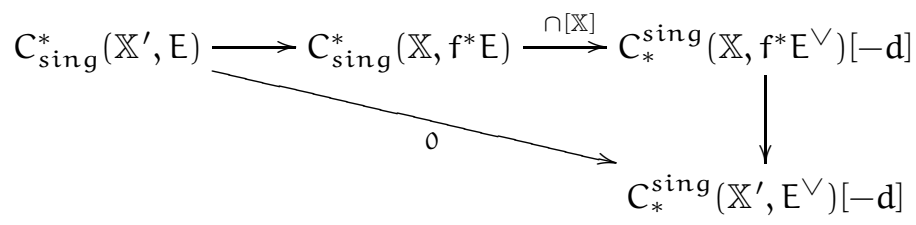

Hence the horizontal composition lifts to a map $C_{\text {sing }}^{*}\left(\mathbb{X}^{\prime}, E\right) \longrightarrow C_{*}^{\text {sing }}\left(f, E^{\vee}\right)[-d-1]$ denoted $\cap\left[\mathbb{X}^{\prime}\right]$, where $C_{*}^{\operatorname{sing}}\left(f, E^{\vee}\right)$ is the homotopy cofiber of $C_{*}^{\text {sing }}\left(\mathbb{X}, f^{*} E^{\vee}\right) \rightarrow C_{*}^{\text {sing }}\left(\mathbb{X}^{\prime}, E^{\vee}\right)$. The non-degeneracy condition can then be restated as follows: $\cap\left[\mathbb{X}^{\prime}\right]$ is an isomorphism in $h C p x$.

Example 4.2. Oriented Poincaré d-pairs provide examples of relative d-orientations.

We now prove a result that will allow us to define composition of cospans between d-oriented spaces.

Theorem 4.3. Let $\mathbb{X}, \mathbb{Y}, \mathbb{Z}$ be three $\mathrm{d}$-oriented spaces and assume we have relative $\mathrm{d}$-orientations on the maps $\mathrm{f}: \mathbb{X} \amalg \overline{\mathbb{Y}} \rightarrow \mathbb{F}$ and $\mathrm{g}: \mathbb{Y} \amalg \overline{\mathbb{Z}} \rightarrow \mathbb{G}$, where $\overline{\mathbb{Y}}$ means that we consider $\mathbb{Y}$ equipped with the opposite fundamental class. Then we have a relative d-orientation on $\mathrm{t}: \mathbb{X} \amalg \overline{\mathbb{Z}} \rightarrow \mathbb{T}:=\mathbb{F} \coprod_{\mathbb{Y}}^{h} \mathbb{G}$.

Proof. Let us write $i$, resp. $j$, for the map $\mathbb{F} \rightarrow \mathbb{T}$, resp. $\mathbb{G} \rightarrow \mathbb{T}$. We first observe that the relative d-orientation on $f$ produces a path from $f_{*}[\mathbb{X}]$ to $f_{*}[\mathbb{Y}]$, and thus from $i_{*} f_{*}[\mathbb{X}]$ to $i_{*} f_{*}[\mathbb{Y}]$. Similarly we have a path from $j_{*} g_{*}[\mathbb{Y}]$ to $j_{*} g_{*}[\mathbb{Z}]$. Finally, there is (by definition of the push-out) a path from $i_{*} f_{*}[\mathbb{Y}]$ to $j_{*} g_{*}[\mathbb{Y}]$. Composing these three paths we get a path from $i_{*} f_{*}[\mathbb{X}]$ to $j_{*} g_{*}[\mathbb{Z}]$, which defines a boundary structure on the morphism $t_{\mathrm{B}}$.

We then have to prove that it is non-degenerate. One way of doing that is by contemplating the following homotopy commuting diagram, in which all rows are exact (and $E$ is a perfect local system):

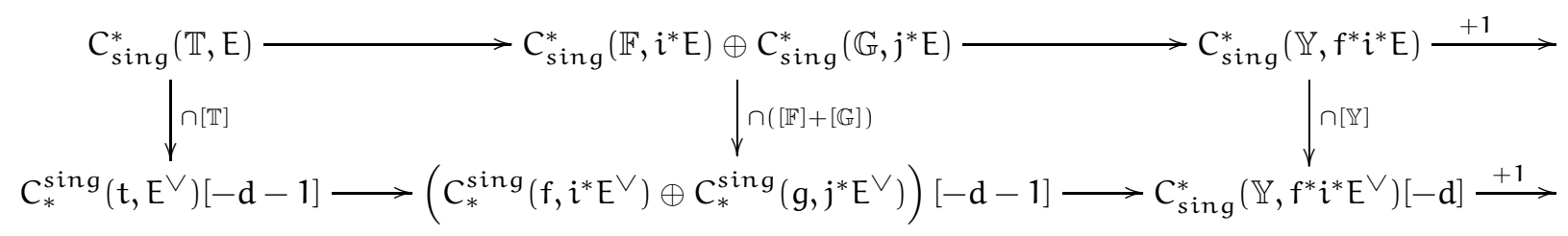

The first vertical arrow is an isomorphism in $h \mathrm{hpx}$ because the last two ones are. 
Let $\operatorname{Cosp}^{\mathrm{d} \text {-or }}$ be the category with objects being d-oriented spaces and Hom Cosp $^{\text {d-or }}(\mathbb{X}, \mathbb{Y})$ being weak equivalence classes of d-oriented maps $\mathbb{X} \coprod \bar{Y} \rightarrow \mathbb{F}$, which we call d-oriented cospans. The composition of two morphisms is given by their homotopy push-out, as in 4.1 .2 , with d-orientation given by Theorem 4.3. It is again symmetric monoidal, with monoidal product $\coprod$.

For every $\mathrm{d} \geq 0$ there is a symmetric monoidal functor $\mathcal{F}^{\text {or }}: \mathrm{dCob}^{\text {or }} \rightarrow$ Cosp ${ }^{\mathrm{d} \text {-or }}$ which sends an oriented d-dimensional differentiable manifold to its underlying d-oriented space and an oriented cobordism of dimension $d+1$ to the corresponding $d$-oriented cospan. It fits into a commuting diagram

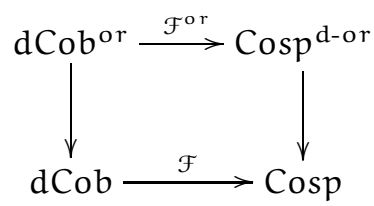

where the vertical arrows consist in forgetting orientations.

\subsubsection{A category of Lagrangian correspondences}

We start with an analog of Theorem 4.3 for Lagrangian structures, which generalizes Theorem 2.4 on derived Lagrangian intersections.

Theorem 4.4. Let $\mathrm{L}_{1} \stackrel{\mathrm{f}_{1}}{\longrightarrow} \mathbf{X} \times \mathbf{Y}$ and $\mathbf{L}_{2} \stackrel{\mathrm{f}_{2}}{\longrightarrow} \mathbf{Y} \times \mathbf{Z}$ be morphisms of derived Artin stacks, and assume that $\mathbf{X}, \mathbf{Y}$ and $\mathbf{Z}$ are equipped with $\mathrm{n}$-symplectic structures $\omega_{\mathbf{X}}, \omega_{\mathbf{Y}}$ and $\boldsymbol{\omega}_{\mathbf{Z}}$. Then there is a map

$$
\operatorname{Lagr}\left(f_{1}, \pi_{X}^{*} \omega_{X}-\pi_{Y}^{*} \omega_{Y}\right) \times \operatorname{Lagr}\left(f_{2}, \pi_{Y}^{*} \omega_{Y}-\pi_{Z}^{*} \omega_{Z}\right) \longrightarrow \operatorname{Lagr}\left(g, \pi_{X}^{*} \omega_{X}-\pi_{Z}^{*} \omega_{Z}\right),
$$

where $\mathrm{g}: \mathrm{L}_{12}:=\mathrm{L}_{1} \underset{\mathrm{Y}}{\stackrel{\mathrm{h}}{\times}} \mathrm{L}_{2} \rightarrow \mathbf{X} \times \mathbf{Z}$.

Proof. The proof is very similar to the one of Theorem 4.3. We first observe that the result we want to prove holds for isotropic structures. Namely, a Lagrangian structure on $f_{1}$ is the same as a path from $f_{1}^{*} \pi_{X}^{*} \omega_{X}$ to $f_{1}^{*} \pi_{Y}^{*} \omega_{Y}$ in $\Omega_{\mathbf{L}_{1}}^{2, c l}$, a Lagrangian structure on $f_{2}$ is the same as a path from $f_{2}^{*} \pi_{Y}^{*} \omega_{Y}$ to $f_{2}^{*} \pi_{Z}^{*} \omega_{Z}$ in $\Omega_{L_{2}}^{2, c l}$, and we have a natural path from $\pi_{L_{1}}^{*} f_{1}^{*} \pi_{Y}^{*} \omega_{Y}$ to $\pi_{L_{2}}^{*} f_{2}^{*} \pi_{Y}^{*} \omega_{Y}$ in $\Omega_{L_{12}}^{2, c l}$. Hence we get a path from $\pi_{L_{1}}^{*} f_{1}^{*} \pi_{X}^{*} \omega_{X}$ to $\pi_{L_{2}}^{*} f_{2}^{*} \pi_{Z}^{*} \omega_{Z}$ in $\Omega_{L_{12}}^{2, c l}$, which defines an isotropic structure on $g$.

We then have to prove that it is non-degenerate. As before, we invite the reader to contemplate a homotopy commuting diagram in which all rows are exact:

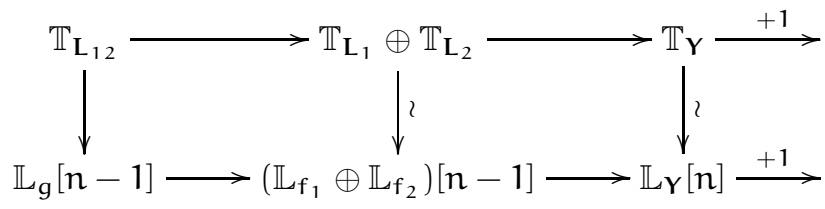

Let LagCorr $n$ be the category with objects being $n$-symplectic derived Artin stacks and Hom Lag Corr $_{n}(X, Y)$ being weak equivalence classes of Lagrangian morphisms $\mathbf{L} \rightarrow \mathbf{X} \times \overline{\mathrm{Y}}$, where $\overline{\mathrm{Y}}$ is $\mathrm{Y}$ endowed with the opposite symplectic structure. The composition of two morphisms is their homotopy fiber product, as in 4.2 .2 . endowed with the Lagrangian structure given by Theorem 4.4.

It again has a symmetric monoidal structure, with monoidal product the Cartesian product.

Example 4.5. Let $\mathrm{f}: \mathrm{X} \rightarrow \mathrm{Y}$ be a morphism between derived $n$-symplectic stacks equiped with a symplectomorphism structure, that is to say a Lagrangian structure on the graph $\mathrm{X} \rightarrow \mathrm{X} \times \mathrm{Y}$, where $\mathrm{X} \times \mathrm{Y}$ is equipped with the difference of the symplectic structures on $\mathrm{X}$ and $\mathrm{Y}$ (i.e. a non-degenerate path from $f^{*} \omega_{Y}$ to $\omega_{X}$ in $\left.\operatorname{Symp}(X, n)\right)$.

Proposition 4.6. For any morphism $\mathrm{g}: \mathrm{L} \rightarrow \mathrm{Y}$ we have a map

$$
\operatorname{Lagr}\left(\mathrm{g}, \omega_{\mathrm{Y}}\right) \longrightarrow \mathbb{L} \operatorname{Lagr}\left(\pi_{1}, \omega_{\mathrm{X}}\right)
$$

where $\pi_{1}: \mathbf{Z}:=\mathbf{X} \underset{\mathrm{Y}}{\stackrel{h}{\times}} \mathbf{L} \rightarrow \mathbf{X}$ is the "first projection". 
Proof. This is a direct corollary of Theorem 4.4 .

Example 4.7. Let $H, G$ be reductive algebraic groups and let $X$ be a quasi-Hamiltonian $\mathrm{G}^{\times 2} \times \mathrm{H}_{\text {-space: }}$ there is an equivariant map $\mathrm{X} \rightarrow \mathrm{G}^{\times 2} \times \mathrm{H}$ such that $\left[\mathrm{X} / \mathrm{G}^{\times 2} \times \mathrm{H}\right] \rightarrow\left[\mathrm{G}^{\times 2} \times \mathrm{H} / \mathrm{G}^{\times 2} \times \mathrm{H}\right]$ has a Lagrangian structure. Observe that the diagonal map $\mathrm{G} \rightarrow \mathrm{G} \times \mathrm{G}$ gives rise to two morphisms

$$
[\mathrm{G} \times \mathrm{G} / \mathrm{G}] \rightarrow[\mathrm{G} / \mathrm{G}] \text { and }[\mathrm{G} \times \mathrm{G} / \mathrm{G}] \rightarrow[\mathrm{G} \times \mathrm{G} / \mathrm{G} \times \mathrm{G}]
$$

One can prove that their product $[\mathrm{G} \times \mathrm{G} / \mathrm{G}] \longrightarrow[\mathrm{G} / \mathrm{G}] \times \overline{[\mathrm{G} \times \mathrm{G} / \mathrm{G} \times \mathrm{G}]}$ carries a Lagrangian structure, and thus so does $\left[\mathrm{G}^{2} \times \mathrm{H} / \mathrm{G} \times \mathrm{H}\right] \longrightarrow[\mathrm{G} \times \mathrm{H} / \mathrm{G} \times \mathrm{H}] \times \overline{\left[\mathrm{G}^{2} \times \mathrm{H} / \mathrm{G}^{2} \times \mathrm{H}\right]}$.

From Theorem 4.4 we get that

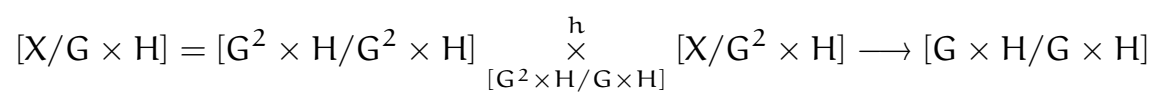

carries a Lagrangian structure. Following [1, Section 6] we call it the internal fusion of X, which consists of a quasi-Hamiltonian structure on the composed map $\mathrm{X} \rightarrow \mathrm{G}^{2} \times \mathrm{H} \rightarrow \mathrm{G} \times \mathrm{H}$. This gives a new interpretation of [1, Theorem 6.1]. We refer to [17] for more details about the relevance of derived symplectic and Lagrangian structures for quasi-Hamiltonian geometry.

\subsubsection{Oriented TFTs with values in Lagrangian correspondences}

We fix an $n$-symplectic stack $(\mathbf{Y}, \omega)$.

Theorem 4.8. The symmetric monoidal functor $\operatorname{Map}\left((-)_{\mathrm{B}}, \mathrm{Y}\right): \operatorname{Cosp} \rightarrow$ Corr can be lifted to a symmetric monoidal functor $\operatorname{Cosp}^{\mathrm{d}-\mathrm{or}} \rightarrow \mathrm{LagCorr}_{\mathrm{n}-\mathrm{d}}$.

As a consequence we get that the functor $z_{Y}: \mathrm{dCob} \rightarrow$ Corr can be lifted to a symmetric monoidal functor $Z_{Y}^{\text {or }}: \mathrm{dCob}^{\text {or }} \rightarrow$ LagCorr $_{n-\mathrm{d}}$, which means that we have a commuting diagram

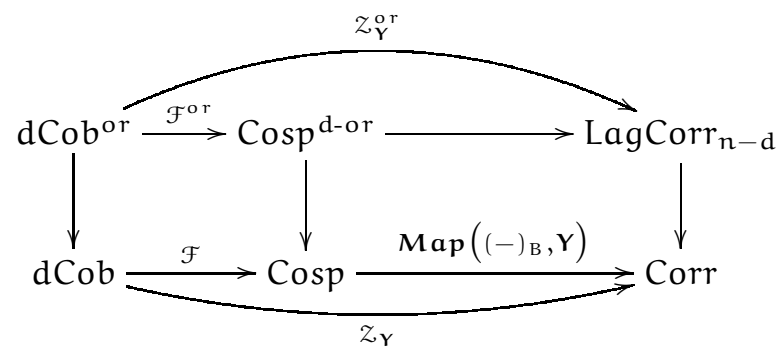

Proof of Theorem 4.8. On objects, if $\mathbb{X}$ is $d$-oriented then by Theorem 1.5 we have an $(n-d)$-symplectic structure on the mapping stack $\operatorname{Map}\left((\mathbb{X})_{\mathrm{B}}, Y\right)$. On morphisms, if $\mathbb{X} \coprod \mathbb{Y} \rightarrow \mathbb{F}$ carries a relative dorientation then by Theorem 2.9 the morphism

$$
\operatorname{Map}\left((\mathbb{F})_{B}, Y\right) \longrightarrow \operatorname{Map}\left((\mathbb{X} \coprod \bar{Y})_{B}, Y\right)=\operatorname{Map}\left((\mathbb{X})_{B}, Y\right) \times \overline{\operatorname{Map}\left((\mathbb{Y})_{B}, Y\right)}
$$

inherits a Lagrangian structure. It is very easy to check from the construction that this is compatible with composition of morphisms.

\section{Concluding remarks}

In a subsequent work we will show how the above constructions can lead to fully extended oriented TFTs in the sense of Baez-Dolan and Lurie (see [3, 12, ), as well as to theorie with boundary and/or defects. We aslo conjecture that our approach could provide a rigorous construction of the 2 dimensional TFT with values in holomorphic symplectic manifolds that has been discovered by Moore-Tachikawa [13].

Below we sketch the construction of semi-classical TFTs with boundary conditions from relative derived mapping stacks (see also [7]). 


\section{TFTs with boundary conditions}

We briefly explain how can one extend our methods to the construction of TFTs in the presence of boundary conditions.

\section{The category of cobordisms with boundary}

One can introduce the category $\mathrm{dCob}_{\mathrm{bnd}}^{(\mathrm{or})}$ with objects being (oriented) compact d-dimensional manifolds with boundary, and morphisms being diffeomorphisms classes of (oriented) $(d+1)$-dimensional cobordisms with boundary. The disjoint union turns it into a symmetric monoidal category. An (oriented) topological field theory with boundary is a symmetric monoidal functor $\mathrm{dCob}_{\mathrm{bnd}}^{(\text {or })} \rightarrow \mathcal{C}$.

\section{An oriented TFT with boundary from relative derived mapping stacks}

If we fix an $n$-symplectic stack and a Lagrangian morphism $\mathrm{f}: \mathrm{L} \rightarrow \mathrm{Y}$ then we can construct a symmetric monoidal functor $Z_{f}^{\text {or }}: \mathrm{dCob}_{\mathrm{bnd}}^{\text {or }} \longrightarrow$ LagCorr $_{\mathrm{n}-\mathrm{d}}$ which does the following on objects: if $\Sigma$ is an oriented compact d-dimensional manifolds with boundary then the inclusion map $\iota_{\Sigma}: \partial \Sigma \rightarrow \Sigma$ is relatively $(d-1)$ oriented and we define $z_{f}^{o r}(\Sigma):=\operatorname{Map}\left(\left(\iota_{\Sigma}\right)_{B}, f\right)$, which naturally carries an $(n-d)$-symplectic structure by Theorem 2.11 .

One way to prove this is first to construct the following three oriented TFTs with boundary:

- $z_{1}: \Sigma \mapsto \operatorname{Map}\left(\Sigma_{\mathrm{B}}, f\right)$, which takes values in a category $\mathcal{C}$ where objects themselves are Lagrangian morphisms (and morphisms are weak equivalence classes of correspondences between them).

- $z_{2}: \Sigma \mapsto \operatorname{Map}\left(\left(\iota_{\Sigma}\right)_{\mathrm{B}}, \mathrm{Y}\right)$, which also takes values in $\mathrm{C}$.

- $Z_{3}: \Sigma \mapsto \operatorname{Map}\left((\partial \Sigma)_{\mathrm{B}}, \mathrm{Y}\right)$, which takes values in $\operatorname{LagCorr}_{\mathrm{n}-\mathrm{d}+1}$.

Then one observes that there is a forgetful functor $\mathcal{F}: \mathcal{C} \rightarrow \operatorname{LagCorr}_{n+1+1}$ and that $\mathcal{F} \circ \mathcal{Z}_{1}=Z_{3}=\mathcal{F} \circ Z_{2}$. Moreover, the derived fiber product of Lagrangian morphisms gives a functor

$$
\mathcal{G}: \underset{\operatorname{LagCorr}_{n-d}+1}{\mathrm{C}} \underset{\operatorname{LagCorr}}{\mathrm{L}-\mathrm{d}}
$$

so that in the end $z_{f}^{o r}=\mathcal{G} \circ\left(z_{1} \times z_{2}\right)$.

The best way to make sense of this is to work with the higher categories of (iterated) Lagrangian correspondences that have been informally introduced at the beginning of the paper.

\section{References}

[1] A. Alekseev, A. Malkin \& E. Meinrenken, Lie Group Valued Moment Maps, J. Differential Geom. 48 (1998), no. 3, 445-495.

[2] M. Alexandrov, M. Kontsevich, A. Schwarz \& O. Zaboronsky, The geometry of the master equation and topological quantum field theory, Int. J. Modern Phys. A12 (1997), no. 7, 1405-1429.

[3] J. Baez \& J. Dolan, Higher dimensional algebra and topological quantum field theory, J. Math. Phys. 36 (1995), 6073-6105.

[4] C. Barwick \& D.M. Kan, Relative categories: Another model for the homotopy theory of homotopy theories, Indagationes Mathematicæ 23 (2012), no. 1-2, 42-68.

[5] I. Batalin \& G.Vilkovisky, Gauge Algebra and Quantization, Phys. Lett. B 102 (1981), no. 1, 27-31.

[6] F. Bottacin, Poisson structures on moduli spaces of framed vector bundles on surfaces, Math. Nachr 220 (2000), 33-44.

[7] D. Calaque, Three lecture on derived symplectic geometry and topological field theories, Indagationes Mathematicæ 25 (2014), no. 5, 926-947, Poisson 2012: Poisson Geometry in Mathematics and Physics.

[8] A. Cattaneo \& G. Felder, On the AKSZ formulation of the Poisson sigma model, Lett. Math. Phys. 56 (2001), 163-179. 
[9] _ Coisotropic submanifolds in Poisson geometry and branes in the Poisson sigma model, Lett. Math. Phys. 69 (2004), 157-175.

[10] A. Cattaneo, P. Mnev \& N. Reshetikhin, Classical BV theories on manifolds with boundary, Communication in Mathematical Physics 332 (2014), 535-603.

[11] _ Classical and quantum Lagrangian field theories with boundary, Proceedings of the "Corfu Summer Institute 2011 School and Workshops on Elementary Particle Physics and Gravity", PoS(CORFU2011)044.

[12] J. Lurie, On the classification of topological field theories, Current developments in mathematics, 2008, 129-280, Int. Press, Somerville, MA, 2009.

[13] G. Moore \& Y. Tachikawa, On 2d TQFTs whose values are holomorphic symplectic varieties, in String-Math 2011, Proceedings of Symposia in Pure Mathematics 85, AMS, 2012.

[14] S. Mukai, Symplectic structure of the moduli space of sheaves on an abelian or K3 surface, Inventiones Mathematicae 77 (1984), no. 1, 101-116.

[15] T. Pantev, B. Toën, M. Vaquié \& G. Vezzosi, Shifted symplectic structures, Publ. Math. IHÉS 117 (2013), no. 1, 271-328.

[16] L. Rozansky \& E. Witten, Hyper-Kähler geometry and invariants of three-manifolds, Selecta Math., New Ser. 3 (1997), no. 3, 401-458.

[17] P. Safronov, Quasi-Hamiltonian reduction via classical Chern-Simons theory, preprint arXiv: 1311.6429

[18] F. Sala, Symplectic structures on moduli spaces of framed sheaves on surfaces, Central European Journal of Mathematics 10 (2012), no. 4, 1455-1471.

[19] B. Toën \& G. Vezzosi, Homotopical algebraic geometry II: geometric stacks and applications, Memoirs of the AMS 193 (2008), no. 2.

[20] _ From HAG to DAG: derived moduli stacks, Axiomatic, Enriched and Motivic Homotopy Theory, NATO Science Series, 2004, Volume 131, Part II, 173-216.

[21] B. Toën, Derived Algebraic Geometry, preprint arXiv:1401.1044, to appear in EMS Surveys in Mathematical Sciences.

[22] A. Tyurin, Fano versus Calabi-Yau, preprint arXiv:math/0302101. 